\title{
UAV-Enabled Cooperative NOMA with Indoor- Outdoor User-Paring and SWIPT in $\kappa-\mu$ Channels
}

\author{
A. Alqahtani, Student Member, IEEE, E. Alsusa, Senior Member, IEEE, A. Al-Dweik, Senior Member, IEEE,
}

\begin{abstract}
This work presents a performance analysis on cooperative non-orthogonal multiple accesses (C-NOMA) when assisted with energy harvesting enabled unmanned aerial vehicle (UAV) decode-and-forward (DF) relaying. In particular, two scenarios are considered, an outdoor-indoor one, where the NOMA signal propagates through outdoor-to-indoor, and a conventional outdoor scenario where the channel gains follow a $\kappa-\mu$ generalized fading model. The objectives of this work is to analyze the downlink performance of this C-NOMA system and derive closed-form expressions for the outage probability (OP), ergodic capacity (EC), throughput and energy efficiency (EE) for the users assuming imperfect successive interference cancellation (SIC). In particular, the OP approach considers the individual users' rate where it is required to satisfy certain quality of service $(Q \circ S)$ requirements. The results provide insights into the considered performance metrics relative to key parameters such as power allocation, power splitting factor, fading parameters, and residual interference. Extensive simulations results are presented to validate the accuracy of the derived expressions.
\end{abstract}

Index Terms-NOMA, OP, EC, $\kappa-\mu$ Fading Model, SWIPT.

\section{INTRODUCTION}

A CCOMMODATING the rapid growth of data-intensive wireless applications requires highly innovative mechanisms to enable efficient utilization of the available radio spectrum while satisfying various quality of service (QoS) metrics such as data rate, latency, outage, and error rate. Although the fifth generation $(5 \mathrm{G})$ of wireless networks was designed to satisfy such QoS requirements [2], [3], the continuous growth is expected to push $5 \mathrm{G}$ to its limits, which motivates the need to look for enhanced technologies beyond 5G. Recently, non-orthogonal multiple accesses (NOMA) has emerged as a potential multiple access technology that can fulfill the future wireless demands [4]. The fundamental concept of NOMA is to multiplex the data of multiple users through superposition coding (SC) at the transmitter while taking into account their channels' conditions. In power domain NOMA, multiple users are allocated the same time and frequency transmission resources, but different power levels. Therefore, users with good channel conditions may be assigned less transmit power, and users with poor channel conditions are allocated more transmit power. While the latter can decode their information directly, users allocated less power may use successive interference cancellation (SIC) to retrieve their information [5].

A. Alqahtani and E. Alsusa are with the School of Electrical and Electronic Engineering, University of Manchester, Manchester M1 3WE, UK (e-mail: adel.alqahtani@postgrad.manchester.ac.uk, e.alsusa@manchester.ac.uk).

A. Al-Dweik is with the C2PS Center, Khalifa University, Abu Dhabi, UAE, and also with the Department of Electrical and Computer Engineering, Western University, London, ON, Canada. (e-mail: dweik@fulbrightmail.org).

This work was published in part at the 2020 IEEE VTC conference [1].

\section{A. Related Work}

There are numerous research studies on NOMA and its applications with other concepts such as cooperative transmission, simultaneous wireless information and power transfer (SWIPT), and unmanned aerial vehicle (UAV) relays. For example, the authors in [6] studied different categories of NOMA focusing on key features and challenges. They also provided fundamental comparisons between NOMA and orthogonal multiple access (OMA), where it is shown that NOMA outperforms OMA in many popular scenarios. In addition, the studies in [7] and [8] investigate the impact of ideal and practical channel state information (CSI) on NOMA systems. In [9], cooperative non-orthogonal multiple accesses (C-NOMA) is proposed where a dedicated relay is applied to enhance the capacity and coverage of NOMA users. A C-NOMA proposed in [10] where a dedicated successive user relaying is used to enhance the spectral efficiency. The results showed that successive relaying techniques outperform conventional half-duplex (HD) schemes. The authors in [11] proposed a number of cooperative schemes such as decodeand-forward (DF) relay, known as fixed relaying (FR), selective DF with coordinated direct and relay transmission (SDFCDRT), and incremental-selective DF (ISDF) relaying. In a study of C-NOMA given in [12], Bariah et al. investigated the error rate performance of NOMA based relay networks with energy harvesting. The results indicate that overall performance is effected by the selection of different impact factors such as power splitting coefficient, relay's location, and allocated transmitting power. In [13], the authors studied the error rate of C-NOMA based cognitive radio networks with partial relay selection, where an exact expression of pairwise error probability is attained to evaluate the BER union bound. Moreover, several research studies have been performed on UAV-relay C-NOMA based systems. For example, the authors in [14] suggested that a UAV-relay flies at a fixed altitude to assist a pair of outdoor NOMA users, and attempted to maximize the sum-rate of the considered system and the harvested energy at the UAV node. The work in [15] proposed an outdated relay selection algorithm for amplify-andforward (AF) relay-assisted C-NOMA, where several UAV's are supported to assist outdoor users. The study investigates the impact of outdated coefficients, the transmission signalto-noise ratio (SNR), the number of relays, and the average channel gain components on the outage performance. On the other hand, NOMA has been combined with other technologies for indoor environments. Unlike [16] and [17], where NOMA users are entirely distributed in outdoor environments, [18] shows the application of NOMA with indoor visible light 
communication (VLC), whereby the users are distributed in an indoor environment. The study demonstrated a remarkable performance gain over OMA, where all the users exhibit realistic conditions with illumination design restrictions.

The impact of user pairing on NOMA has been widely investigated in the literature. For instance, the authors in [17] studied the impact of user pairing on two NOMA users by analyzing the sum-rate of two different NOMA schemes known as fixed NOMA (F-NOMA) and cognitive radio NOMA (CRNOMA) systems. For F-NOMA, the authors proposed to select a pair of users whose channel conditions are distinctly different. However, for CR-NOMA, the user pairing process mainly depends on the condition to prioritize the primary user's QoS and/or fairness. A further study on NOMA user pairing, where the targeted users are randomly located in a cooperative network with SWIPT, is presented in [19]. The authors proposed three different techniques of user selections based on the user distance from the base station. These are known as follows: random near user and random far user (RNRF) pairing, nearest near user and nearest far user (NNNF) pairing, and nearest near user and farthest far user (NNFF) pairing. The results disclose that NNFF outperforms other techniques in terms of low outage and high throughput for both near and far users. Moreover, other recent works, restricted to outdoor-to-outdoor channel deployment, introduced some advanced algorithms in order to efficiently solve the assignment problem of NOMA users. To illustrate, in [20], the authors propose two user pairing algorithms for cooperative NOMA with SWIPT applications. These algorithms are mainly attained to minimizing the inter-pair interference of the far user caused by zero-forcing beam-forming. In addition, a power allocation scheme is also proposed to guarantee the users' performance. In the case of multiple NOMA users, the study in [21] aims to improve the spectral efficiency, data rate, fairness, and outage probability of multiple NOMA users by proposing two sub-carrier user assignment algorithms (SUAAs). The pairing procedure of both algorithms is predominantly determined by increasing the channel conditions of the designated paired users per subcarrier to enhance the data rate of each individual user. The results of that work show significant improvements over other proposed algorithms by obtaining high spectral efficiency, data rate, and outage probability performance. Another study on user pairing that considered NOMA based UAV system is presented in [22]. The authors optimized the power allocation, user pairing, and UAV placement in order to maximize the minimum sum rate for each selected user pairs. The results illustrate the key significance of UAV placement optimization, where the proposed heuristic user pairing algorithm offers close performance to the optimal solution.

\section{B. Motivation and Contribution}

In the aforementioned studies, the considered NOMA signals were considered to propagate through outdoor-to-outdoor or indoor-to-indoor channels, while often users can be indoor while they are served by an outdoor base station (BS). As such, the outdoor-to-indoor channels, or vise versa, are an important consideration that requires further investigation by the research community. In fact, to the best of the authors' knowledge, no research work has been carried out on this scenario which enables pairing users with roughly the same distances, but different attenuation factors due to being in different environments. Although the open literature presents several studies on conventional outdoor NOMA, it is not directly applicable for the case of indoor NOMA. Motivated by this, we aim to study two different scenarios for NOMA users where the downlink superimposed signal propagates through outdoor-to-indoor and outdoor-to-outdoor channels. Specifically, we provide analytical evaluation of the C-NOMA performance for both cases by considering the instantaneous channels' gains of all the available links, i.e., the product of distance-dependent path-loss and the channel fading gain. The contributions of this paper can be summarized as follows:

- Derive the signal to interference plus noise ratio (SINR) of both users to find exact outage probability (OP) expressions for the indoor/outdoor NOMA users over a generalized $\kappa-\mu$ fading channel when the user's demand of data rates are dropped below certain QoS requirements.

- With the aid of the Fox H-function, derive exact analytical expression for the ergodic capacity (EC). In addition, closed-forms expressions are obtained for the throughput and energy efficiency (EE) of a two-user NOMA system under ideal and practical assumptions.

- Evaluate the performance of downlink NOMA signals propagating through outdoor-to-indoor and outdoor-tooutdoor channels, where both channels are ranked based on their instantaneous fading coefficients.

- Discuss the the path-loss characteristics of the indoor and outdoor users, since NOMA users are ranked according to the product of their distance-dependent path-loss and channel fading gains.

- Extend the system model to include DF UAV-relay with HD mode, and SWIPT with time switching-based relaying (TSR) protocol and consider practical issues such imperfect SIC $(\eta)$ in all the derivations.

- Validate the accuracy of the derived expressions using Monte Carlo Simulations.

\section{Paper Organization}

The rest of this article is organized as follows. Section II presents the system model of this work which includes subsections of channel characteristics, path loss model, and fading distributions. Sections III provides the various performance metrics such as SINR, OP, EC, EE and throughput, while the results are discussed in section $\mathrm{V}$. The conclusions of this work are provided in section VI.

\section{SySTEM MODEL}

This work considers a communications system in which a UAV-relay is deployed to assist the communications between a terrestrial BS and two far NOMA users deployed in two environments, an indoor user $(\mathcal{I})$, and an outdoor user $(\mathcal{O})$. This system model can exist when both users are beyond the coverage of the BS due to large obstacles in the path of the signal, or in an emergency case, where the ground infrastructures 


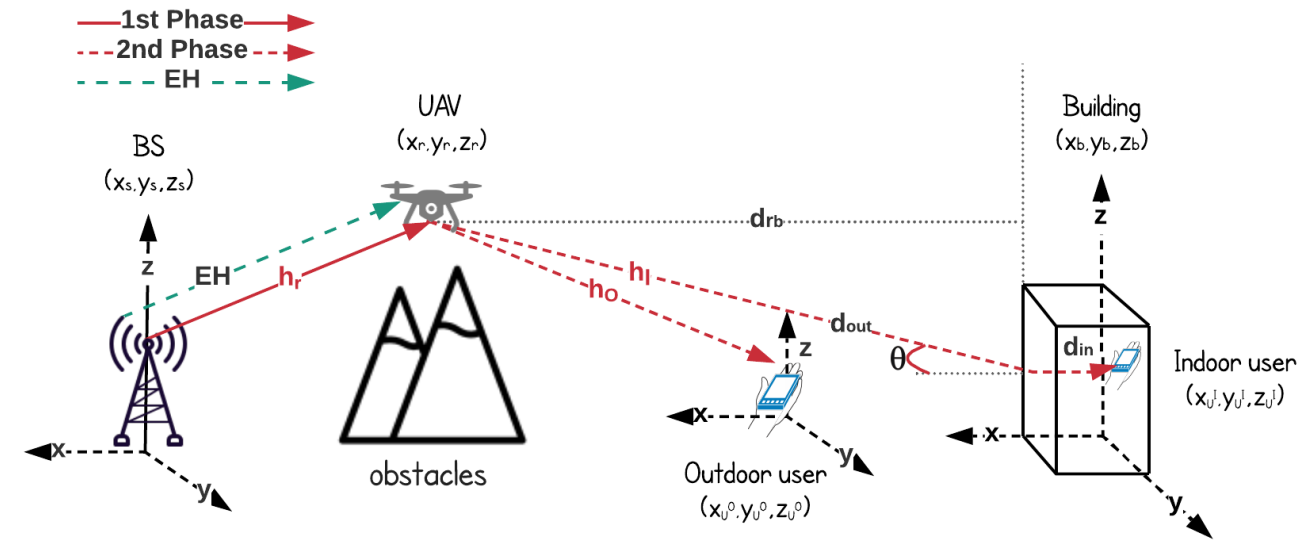

Fig. 1. System Model Under consideration with indoor and outdoor user-pairing.

are hampered due to a natural event such as an earthquake. All terminals are assumed to be equipped with a single antenna, and the whole transmission process is performed in two different time slots, where the relay node applies the HD protocol. As depicted in Fig. 1, the intention is to concentrate on the downlink scenario of the C-NOMA system. We also assume that $\mathcal{I}$ is located inside a building that has location coordinates identified by $\left(x_{b}, y_{b}, z_{b}\right)$. Similarly, each assigned user is located at different coordinates known as $\left(x_{i}, y_{i}, z_{i}\right)$, where $i \in\{\mathcal{I}, \mathcal{O}\}$. In addition, the location coordinates for the $\mathrm{BS}$ and the UAV-relay are $\left(x_{s}, y_{s}, z_{s}\right)$, and $\left(x_{r}, y_{r}, z_{r}\right)$, respectively. Thus, the distances between the BS and UAV can be calculated as $d_{\mathrm{sr}}=\sqrt{\left(x_{s}-x_{r}\right)^{2}+\left(y_{s}-y_{r}\right)^{2}+\left(z_{s}-z_{r}\right)^{2}}$. Similarly, the distance between the UAV and user $i$ is given by $d_{\mathrm{ri}}=\sqrt{\left(x_{r}-x_{i}\right)^{2}+\left(y_{r}-y_{i}\right)^{2}+\left(z_{r}-z_{i}\right)^{2}}$.

\section{A. Channel and Path Loss Models}

Without loss of generality, we assume that all adopted wireless channels are independent and identically distributed (i.i.d) and follow the general $\kappa-\mu$ fading model [23]. It is also assumed that all CSI are available at BS. Without loss of generality, the channel coefficients between the BS and all the nodes are considered to be in ascending order as $\left|h_{\mathcal{I}}\right| \leq\left|h_{\mathcal{O}}\right| \leq\left|h_{r}\right|$, where the effects of path loss $(P L)$ and small scale fading $\left(g_{i}\right)$ are given as $\left|h_{i}\right|=\frac{g_{i}}{P L_{i}}$ and $i \in\{\mathcal{I}, \mathcal{O}$, $r\}$, [24]. Particularly, $g_{i}$ is modeled as $\kappa-\mu$ fading in this work. According to Fig. 1, the NOMA signal is transported over two transmission phases via a UAV-relay, operated in HD mode, to reach both users. The first phase is established between the air-to-air channel, i.e, $h_{r}$, which only has the impact of a basic outdoor transmission loss, where the transmitted signal attains high opportunity of working under a line of sight (LOS) scenario. This particular large scale path loss is assumed to follow the free space path loss model given in [25] as

$$
P L_{r}=32.45+20 \log _{10}\left(d_{s r}\right)+20 \log _{10}(f)
$$

where $d_{s r}$ is the Euclidean distance between the BS and UAVrelay nodes, and $f$ is the frequency. On the other hand, the second phase occurs over the air-to-ground channels, i.e, $h_{\mathcal{I}}$ and $h_{\mathcal{O}}$, where $\mathcal{O}$ is assumed to have a LOS path with a UAVrelay, which causes only outdoor transmission loss. Thus, the transmission loss between the UAV and $\mathcal{O}$ is described by the free-space path-loss model as

$$
P L_{\mathcal{O}}=32.45+20 \log _{10}\left(d_{r \mathcal{O}}\right)+20 \log _{10}(f) .
$$

The signal fading from the UAV to $\mathcal{I}$ exhibits the outdoorto-indoor path-loss. We assume that the building's wall has a LOS with the transmitting antenna of the UAV. We apply the Building Penetration Loss COST 231 empirical propagation model [25]. This particular model can efficiently describe the propagation path-loss for the indoor user by considering two major transmission path-losses characterized by large scale path-loss and the indoor transmission path-loss, which includes the building penetration loss and the indoor propagation loss. Thus, the total path-loss for $\mathcal{I}$ can be calculated as

$$
P L_{\mathcal{I}}=L_{f s}+L_{t}+L_{i n}
$$

where $L_{f s}$ indicates the free space path-loss. It can be given as in (1) with respect to the measured distance of $\left(d_{r b}+d_{i n}\right)$, where $d_{i n}$ is the distance between the building wall and $\mathcal{I}$, and $d_{r \mathcal{I}}$ is the distance between the UAV and the external building wall. In addition, $L_{t}$ is defined as the transition propagation path-loss that includes the perpendicular loss $L_{p c}$ and the parallel penetration loss $L_{p t}$. It can be presented as

$$
L_{t}=L_{p c}+L_{p t} \times(1-\sin (\theta))^{2}
$$

where $\theta$ is the grazing angle between the UAV and the exterior building wall, which can be given as $\theta=\cos ^{-1}\left(d_{r b} / d_{\text {out }}\right)$. In addition, the indoor propagation path-loss, $L_{i n}$, is defined by the mean path-loss determined from the internal side of the exterior wall, which can be computed as

$$
L_{\text {in }}=\max \left\{n L_{i}, \chi\left(d_{\text {in }}-2\right)(1-\sin (\theta))^{2}\right\}
$$

where $n$ is the number of internal walls, $L_{i}$ defines the loss in the internal walls, and $\chi$ is the indoor path-loss parameter. Typical values for this model parameters are given in table II. 
In this paper, we assume that the link between UAV and $\mathcal{I}$ behaves mostly as non-line of sight (NLOS) since the signal must pass through the wall, so we believe that signal fading is compatible mostly with Rayleigh fading. It can be attained as a special case of the $\kappa-\mu$ generalized fading. We also attempt to test the $\mathcal{I}$ under various fading parameters of $\kappa$ - $\mu$ channel to obtain more insights.

\section{B. Fading Channel Distribution}

The $\kappa-\mu$ distribution represents the small-scale variations of the fading signals in a LOS environment. The signal of $\kappa$ $\mu$ fading constitutes different clusters, $(\mu>0)$, of multipath and scattered waves of identical power, as well as a dominant arbitrary power component constructed inside each cluster. Moreover, the rate between these total components of power is indicated as $(\kappa>0)$. The generalized $\kappa$ - $\mu$ fading model constitutes other well known vital distributions as special cases, such as the One-Sided Gaussian, $(\kappa \rightarrow 0, \mu=0.5)$, the Rayleigh $(\kappa \rightarrow 0, \mu=1)$, the Rice (Nakagami-n) $(\kappa=K$, $\mu=1)$, and the Nakagami-m $(\kappa \rightarrow 0, \mu=m)$. Furthermore, the probability density function (PDF) of the instantaneous SNR of the $\kappa-\mu$ fading channel is given by [26],

$$
\begin{aligned}
f_{\gamma}(\gamma) & =\frac{\mu(1+\kappa)^{\left(\frac{\mu+1}{2}\right)} \gamma^{\left(\frac{\mu-1}{2}\right)}}{\kappa^{\left(\frac{\mu-1}{2}\right)} \mathrm{e}^{(\mu \kappa)} \tilde{\gamma}^{\left(\frac{\mu+1}{2}\right)}} \exp \left(\frac{-\mu(1+\kappa) \gamma}{\tilde{\gamma}}\right) \\
& \times \mathrm{I}_{\mu-1}\left(2 \mu \sqrt{\frac{\kappa(1+\kappa) \gamma}{\tilde{\gamma}}}\right)
\end{aligned}
$$

where the instantaneous SNR is given as $\gamma \triangleq \rho|h|=\frac{\mathcal{P}}{\sigma^{2}}|h|$, and $\rho$ represents the transmitted SNR, $h$ indicates the channel frequency response, $\mathcal{P}$ is the transmit power. In addition, the average SNR can be demonstrated as $\tilde{\gamma} \triangleq \mathbb{E}[\gamma]=\Omega \frac{\mathcal{P}}{\sigma^{2}}$ with $\mathbb{E}[$.] denoting expectation and $\Omega$ defines the mean power of $h$, i.e, $\mathbb{E}|\Omega|=\left[|h|^{2}\right] . \mathrm{I}_{v}($.$) is the modified Bessel function of the$ first kind. To produce a more tractable analysis, we simplify (6) to a further equivalent representation, precisely for $\mathrm{I}_{v}($.$) ,$ by employing the series representation of $\mathrm{I}_{v}($.$) given in [27$, 8.445]. Therefore, (6) is rewritten as

$$
\begin{aligned}
f_{\gamma}(\gamma) & =\frac{1}{\mathrm{e}^{(\mu \kappa)}} \sum_{n=0}^{\infty} \frac{\mu^{2 n+\mu} \kappa^{n}(1+\kappa)^{n+\mu}}{n ! \Gamma(n+\mu) \tilde{\gamma}^{(n+\mu)}} \\
& \times \gamma^{(n+\mu-1)} \exp \left(-\frac{\gamma \mu(1+\kappa)}{\tilde{\gamma}}\right) .
\end{aligned}
$$

The corresponding cumulative density function (CDF) of the $\kappa-\mu$ fading channel is given as [26],

$$
F_{\gamma}(\gamma)=1-\mathcal{Q}_{\mu}[\sqrt{2 \kappa \mu}, \sqrt{2((1+\kappa) \mu \gamma}]
$$

where $\mathcal{Q}_{(.)}$is the generalized Marcum- $Q$ function. Hence, an alternative representation of (8) is obtained by integrating (7) and recalling the identity in [27, 3.381.3]. Therefore, the corresponding CDF is given as

$$
F_{\gamma}(\gamma)=\frac{1}{\mathrm{e}^{(\mu \kappa)}} \sum_{n=0}^{\infty} \frac{(\kappa \mu)^{n}}{n ! \Gamma(n+\mu)} \gamma_{i n c}\left(n+\mu, \frac{\mu(1+\kappa) \gamma}{\tilde{\gamma}}\right)
$$

\section{PERformance AnAlysis}

\section{A. Analysis of SINR}

It is assumed that a fixed power allocation technique is applied in this work where $\alpha_{j}, \forall j \in\{1,2\}$, is subjected to $\alpha_{1}+\alpha_{2}=1$ and $\alpha_{2}<\alpha_{1}$. In addition, $\alpha_{1}$ and $\alpha_{2}$ are respectively indicated for the power allocation factors of $\mathcal{I}$ and $\mathcal{O}$. In the first time slot, the received observation at the UAV can be expressed as

$$
y_{r}=h_{r} \times \underbrace{\left(\sqrt{\alpha_{1} \mathcal{P}_{\mathrm{s}}} x_{1}+\sqrt{\alpha_{2} \mathcal{P}_{\mathrm{s}}} x_{2}\right)}_{x_{s c}}+n_{r}
$$

where $\mathcal{P}_{\mathrm{S}}$ is the total transmitted power at $\mathrm{BS}$, and $x_{s c}$ represents the superposed signal in the first time slot where $x_{1}$ and $x_{2}$ stand for the information symbols of $\mathcal{I}$ and $\mathcal{O}$, respectively, $n_{r}$ indicates the additive white Gaussian noise (AWGN), where $n_{r} \sim \mathcal{C N}\left(0, \sigma_{r}^{2}\right)$. The noise power of both users is assumed to be identical in this work. In the first transmission phase, the UAV-relay's node attempts to detect and decode the received signal for both users by performing SIC. Thereafter, it regenerates a new superimposed signal scaled up with the harvested energy gained from the BS, and forwards it to the nominated users as a second phase of communication. Due to the time splitting $(\tau)$ design at the UAV-relay, the received signal in (10) is divided into two segments according to the TSR protocol; one segment is aimed at energy harvesting $(\mathrm{EH})$, and the other is used for information process (IP) which can be given as

$$
\begin{gathered}
y_{r}^{\mathrm{EH}}=h_{r} \times \underbrace{\left(\sqrt{\alpha_{1} \tau \mathcal{P}_{\mathrm{s}}} x_{1}+\sqrt{\alpha_{2} \tau \mathcal{P}_{\mathrm{s}}} x_{2}\right)}_{x_{s c}(1)}+n_{r} . \\
y_{r}^{\mathrm{IP}}=h_{r} \times \underbrace{\left(\sqrt{\alpha_{1}(1-\tau) \mathcal{P}_{\mathrm{s}}} x_{1}+\sqrt{\alpha_{2}(1-\tau) \mathcal{P}_{\mathrm{s}}} x_{2}\right)}_{x_{s c}(2)}+n_{r} .
\end{gathered}
$$

In order to enhance the user fairness, conventional power allocation is proposed in both time slots, i.e., $\alpha_{1}>\alpha_{2}$, where $\alpha_{1}+\alpha_{2}=1$. Since more power is assigned to the $\mathcal{I}$ symbol, the relay in the first time slot initially detects $x_{1}$ symbol by treating $x_{2}$ symbol as unknown additive noise. Therefore, the maximum likelihood (ML) detection of the $x_{1}$ symbol at the relay is given as

$$
\hat{x}_{1}=\arg \min _{x_{1} \in \mathbb{S}}\left|y_{r}^{I P}-\sqrt{\alpha_{1}(1-\tau) \mathcal{P}_{\mathrm{s}}} h_{r} x_{1}\right|^{2}
$$

where the estimated symbol $\hat{x}_{1}$ belongs to the set $\mathbb{S}$ of all possible constellation points for $\mathcal{I}$. Therefore, the SINR at the UAV-relay to detect $x_{1}$ can be expressed as

$$
\gamma_{r}^{x_{1}}=\frac{\alpha_{1}(1-\tau) \mathcal{P}_{\mathrm{s}}\left|h_{r}\right|^{2}}{\alpha_{2}(1-\tau) \mathcal{P}_{\mathrm{s}}\left|h_{r}\right|^{2}+\sigma_{r}^{2}}=\frac{\alpha_{1} \gamma_{r}}{\alpha_{2} \gamma_{r}+1}
$$

where $\gamma_{r} \triangleq \rho_{r}\left|h_{r}\right|^{2}$ and $\rho_{r}=\frac{(1-\tau) \mathcal{P}_{\mathrm{s}}}{\sigma_{r}^{2}}$, which is the transmitted SNR for the link between BS and UAV.

On the other hand, SIC is applied at the relay to detect $x_{2}$. Therefore, the ML detection of symbol $x_{2}$ for $\mathcal{O}$ is given as

$$
\hat{x}_{2}=\arg \min _{x_{2} \in \mathbb{S}}\left|\hat{y}_{r}^{\mathrm{IP}}-\sqrt{\alpha_{2}(1-\tau) \mathcal{P}_{\mathrm{s}}} h_{r} x_{2}\right|^{2}
$$


where $\hat{x}_{2}$ is the estimated symbol of $\mathcal{O}$ and $y_{r}^{\mathrm{IP}}=$ $\left(y_{r}^{\mathrm{IP}}-\sqrt{\alpha_{1}(1-\tau) \mathcal{P}_{\mathrm{s}}} h_{r} x_{1}\right)$. It is readily apparent that the remaining residual signal after applying SIC mainly relies on the detection of the symbol $x_{1}$. Therefore, the SINR at the UAV-relay to detect $x_{2}$ can be given as

$$
\gamma_{r}^{x_{2}}=\frac{\alpha_{2}(1-\tau) \mathcal{P}_{\mathrm{s}}\left|h_{r}\right|^{2}}{\eta \alpha_{1}(1-\tau) \mathcal{P}_{\mathrm{s}}\left|h_{r}\right|^{2}+\sigma_{r}^{2}}=\frac{\alpha_{2} \gamma_{r}}{\eta \alpha_{1} \gamma_{r}+1}
$$

where the variable $\eta$ indicates the effect coefficient of the incorrect SIC, where $\eta=0$ represents the case of perfect SIC and $\eta=1$ represents the case of completely failed SIC.

The TSR protocol is supposed to split the received superimposed signal, $y_{r}$, arrived at the UAV-relay node, into harvested energy and processed information based on certain time intervals. Therefore, the total time interval for the entire frame $(T)$ should be divided into two main parts. One for the duration of energy harvesting at the UAV-relay indicated as $(\tau T)$, where $0<\tau<1$ is the scaling factor for time division, and the remaining duration, i.e $\frac{(1-\tau) T}{2}$, is evenly distributed between the information processing and energy transmission for the second phase. By performing such a protocol to deploy $\mathrm{EH}$ at the UAV node, the harvested energy amount can be calculated as [28],

$$
E_{h_{r}}^{(\mathrm{TSR})}=\delta \mathcal{P}_{\mathrm{s}}\left|h_{r}\right|^{2} \tau T
$$

where $(0<\delta<1)$ is the energy conversion efficiency. The UAV-relay transmission power $\left(\mathcal{P}_{\mathrm{r}}\right)$ can be found as

$$
\mathcal{P}_{\mathrm{r}}=\frac{E_{h_{r}}^{(\mathrm{TSR})}}{\frac{(1-\tau) T}{2}}=\frac{2 \delta \mathcal{P}_{\mathrm{s}} \tau\left|h_{r}\right|^{2}}{1-\tau}
$$

In the second time slot, the UAV-relay forwards the decoded signal to both users destinations. Therefore, the received signal at the indoor user $\mathcal{I}$ can be expressed as

$$
y^{\mathcal{I}}=h_{\mathcal{I}} \times \underbrace{\left(\sqrt{\alpha_{1} \mathcal{P}_{\mathrm{r}}} x_{1}+\sqrt{\alpha_{2} \mathcal{P}_{\mathrm{r}}} x_{2}\right)}_{x_{s c}(3)}+n_{\mathcal{I}}
$$

where $\mathcal{P}_{\mathrm{r}}$ is the new total transmitted power at the UAV, and $x_{s c}{ }^{(2)}$ represents the imposed signal at the second time slot, where $x_{1}$ and $x_{2}$ refer to the $\mathcal{I}$ and $\mathcal{O}$ information, respectively. $n_{\mathcal{I}}$ indicates the AWGN, where $n_{\mathcal{I}} \sim \mathcal{C N}\left(0, \sigma_{\mathcal{I}}^{2}\right)$. In particular, it is assumed that $\mathcal{O}$ is communicating with the UAV-relay in better channel conditions than the indoor user $\mathcal{I}$, hence $\mathcal{O}$ is allocated less power. Consequently, $\mathcal{I}$ detects its own symbol, $x_{1}$, by considering the $x_{2}$ symbol as unknown additive noise. Hence, the ML detection at $\mathcal{I}$ is expressed as

$$
\hat{x}_{1}=\arg \min _{x_{1} \in \mathbb{S}}\left|y^{\mathcal{I}}-\sqrt{\alpha_{1}(1-\tau) \mathcal{P}_{\mathrm{r}}} h_{\mathcal{I}} x_{1}\right|^{2} .
$$

Therefore, the SINR of $\mathcal{I}$, in the second time slot, to detect the $x_{1}$ symbol can be calculated as

$$
\gamma_{U^{I}}^{x_{1}}=\frac{\alpha_{1} \mathcal{P}_{\mathrm{r}}\left|h_{\mathcal{I}}\right|^{2}}{\alpha_{2} \mathcal{P}_{\mathrm{r}}\left|h_{\mathcal{I}}\right|^{2}+\sigma_{\mathcal{I}}^{2}}=\frac{\alpha_{1} \gamma_{\mathcal{I}}}{\alpha_{2} \gamma_{\mathcal{I}}+1}
$$

where $\gamma_{\mathcal{I}} \triangleq \rho_{\mathcal{I}}\left|h_{\mathcal{I}}\right|^{2}$ and $\rho_{\mathcal{I}} \triangleq \mathcal{P}_{\mathrm{r}} / \sigma_{\mathcal{I}}^{2}$ is the SNR of the link between the UAV and $\mathcal{I}$. On the other hand, the received signal at the $\mathcal{O}$ 's node is expressed as

$$
y^{\mathcal{O}}=h_{\mathcal{O}} \times \underbrace{\left(\sqrt{\alpha_{1} \mathcal{P}_{\mathrm{r}}} x_{1}+\sqrt{\alpha_{2} \mathcal{P}_{\mathrm{r}}} x_{2}\right)}_{x_{s c}(4)}+n_{\mathcal{O}} .
$$

And the corresponding ML detection of $x_{1}$ symbol at $\mathcal{O}$ is

$$
\hat{x}_{1}=\arg \min _{x_{1} \in \mathbb{S}}\left|y^{\mathcal{O}}-\sqrt{\alpha_{1}(1-\tau) \mathcal{P}_{\mathrm{r}}} h_{\mathcal{O}} x_{1}\right|^{2}
$$

Thus, the SINR of $\mathcal{O}$ to detect the $x_{1}$ symbol at the first level of the SIC process, can be given as

$$
\gamma_{\mathcal{O}}^{x_{1} \rightarrow x_{2}}=\frac{\alpha_{1} \mathcal{P}_{\mathrm{r}}\left|h_{\mathcal{O}}\right|^{2}}{\alpha_{2} \mathcal{P}_{\mathrm{r}}\left|h_{\mathcal{O}}\right|^{2}+\sigma_{\mathcal{O}}^{2}}=\frac{\alpha_{1} \gamma_{\mathcal{O}}}{\alpha_{2} \gamma_{\mathcal{O}}+1} .
$$

Similarly, the ML detection for the $x_{2}$ symbol at the same destination can also be written as

$$
\hat{x}_{2}=\arg \min _{x_{2} \in \mathbb{S}}\left|y^{\mathcal{O}}-\sqrt{\alpha_{2}(1-\tau) \mathcal{P}_{\mathrm{r}}} h_{\mathcal{O}} x_{2}\right|^{2}
$$

where $\dot{y}^{\mathcal{O}}=\left(y^{\mathcal{O}}-\sqrt{\alpha_{1}(1-\tau) \mathcal{P}_{\mathrm{s}}} h_{\mathcal{O}} x_{1}\right)$. Therefore, the corresponding SINR to detect $x_{2}$ at $\mathcal{O}$ is defined as

$$
\gamma_{\mathcal{O}}^{x_{2}}=\frac{\alpha_{2} \mathcal{P}_{\mathrm{r}}\left|h_{\mathcal{O}}\right|^{2}}{\eta\left|h_{\mathcal{O}}\right|^{2} \alpha_{1} \mathcal{P}_{\mathrm{r}}+\sigma_{u_{2}}^{2}}=\frac{\alpha_{2} \gamma_{\mathcal{O}}}{\eta \alpha_{1} \gamma_{\mathcal{O}}+1} \text {. }
$$

where $\gamma_{\mathcal{O}} \triangleq \rho_{\mathcal{O}}\left|h_{\mathcal{O}}\right|^{2}$ and $\rho_{\mathcal{O}} \triangleq \mathcal{P}_{\mathrm{r}} / \sigma_{\mathcal{O}}^{2}$ is the SNR of the link between the UAV and $\mathcal{O}$.

\section{B. OP of The Indoor User:}

The outage probability of $\mathcal{I}$ that uses a UAV-relay in the absence of a direct link from BS can be addressed as follows.

\section{Proposition 1.}

$$
\begin{aligned}
\mathrm{P}^{\mathcal{I}} & =1-\prod_{i=1}^{2}\left[1-\frac{1}{\mathrm{e}^{(\mu \kappa)}} \sum_{n=0}^{\infty} \frac{(\kappa \mu)^{n}}{n ! \Gamma(n+\mu)}\right. \\
& \left.\gamma_{\text {inc }}\left(n+\mu, \frac{\mu(1+\kappa) \varepsilon_{1, i}}{\tilde{\gamma}_{1, i}}\right)\right]
\end{aligned}
$$

where $\gamma_{i n c}$ is the incomplete gamma function.

Proof. The outage events of the indoor user $\mathcal{I}$ occur if the following are satisfied. First, when the UAV-relay decodes the signal $x_{1}$ in the first time slot incorrectly. Second, when $\mathcal{I}$ fails in decoding its own signal in the second time slot. Therefore, the outage probability of $\mathcal{I}$ can be expressed as

$$
\begin{aligned}
\mathrm{P}^{\mathcal{I}} & =\mathcal{P}_{\mathrm{r}}\left(\gamma_{r}^{x_{1}}<\gamma_{t h_{1}}, \gamma_{\mathcal{I}}^{x_{1}}<\gamma_{t h_{1}}\right) \\
& =1-\underbrace{\mathcal{P}_{\mathrm{r}}\left(\gamma_{r}^{x_{1}}>\gamma_{t h_{1}}\right)}_{E_{11}} \underbrace{\mathcal{P}_{\mathrm{r}}\left(\gamma_{\mathcal{I}}^{x_{1}}>\gamma_{t h_{1}}\right)}_{E_{12}}
\end{aligned}
$$

where $\gamma_{t h_{1}}=2^{2 R_{\mathcal{I}}}-1$, and $R_{\mathcal{I}}$ is the target data rate of $\mathcal{I}$. Thus, by substituting (14) into $E_{11}$, the following is obtained,

$$
\begin{aligned}
E_{11} & =\mathcal{P}_{\mathrm{r}}\left(\gamma_{r}^{x_{1}}>\gamma_{t h_{1}}\right) \\
& =\mathcal{P}_{\mathrm{r}}\left(\gamma_{0}>\frac{\gamma_{t h_{1}}}{\left(\alpha_{1}-\alpha_{2} \gamma_{t h_{1}}\right)} \triangleq \varepsilon_{1,1}\right) \\
& =1-F_{\gamma}\left(\varepsilon_{1,1}\right) .
\end{aligned}
$$


By substituting (9) in (29) to compute the first term of (28), we obtain the following

$$
E_{11}=1-\frac{1}{\mathrm{e}^{(\mu \kappa)}} \sum_{n=0}^{\infty} \psi \gamma_{i n c}\left(n+\mu, \frac{\mu(1+\kappa) \varepsilon_{1,1}}{\tilde{\gamma}_{1,1}}\right)
$$

where $\psi=\frac{(\kappa \mu)^{n}}{n ! \Gamma(n+\mu)}$, and $\tilde{\gamma}_{1,1}$ indicates the average power link between BS and the UAV-relay. Similarly, $E_{12}$ in (28) can be evaluated by recalling (21) and (9) as follows,

$$
\begin{aligned}
E_{12} & =\mathcal{P}_{\mathrm{r}}\left(\gamma_{\mathcal{I}}^{x_{1}}>\gamma_{t h_{1}}\right) \\
& =\mathcal{P}_{\mathrm{r}}\left(\gamma_{1}>\frac{\gamma_{t h_{1}}}{\left(\alpha_{1}-\alpha_{2} \gamma_{t h_{1}}\right)} \triangleq \varepsilon_{1,2}\right) \\
& =1-F_{\gamma}\left(\varepsilon_{1,2}\right) .
\end{aligned}
$$

Therefore, (31) is regenerated as

$$
E_{12}=1-\frac{1}{\mathrm{e}^{(\mu \kappa)}} \sum_{n=0}^{\infty} \psi \gamma_{i n c}\left(n+\mu, \frac{\mu(1+\kappa) \varepsilon_{1,2}}{\tilde{\gamma}_{1,2}}\right)
$$

where $\tilde{\gamma}_{1,2}$ is the average power link between the UAV-relay and $\mathcal{I}$. By substituting (30) and (32) in (28), in order to obtain proposition 1 . Hence, the proof is complete.

\section{OP of the Outdoor User}

The outage probability of $\mathcal{O}$ when using UAV-relay over $\kappa-\mu$ fading channels can be calculated as follows.

\section{Proposition 2.}

$$
\begin{gathered}
\mathrm{P}^{\mathcal{O}}=1-\prod_{i=1}^{4}\left[1-\frac{1}{\mathrm{e}^{(\mu \kappa)}} \sum_{n=0}^{\infty} \frac{(\kappa \mu)^{n}}{n ! \Gamma(n+\mu)}\right. \\
\left.\gamma_{i n c}\left(n+\mu, \frac{\mu(1+\kappa) \varepsilon_{2, i}}{\tilde{\gamma}_{2, i}}\right)\right]
\end{gathered}
$$

where $\varepsilon_{2,1}, \varepsilon_{2,2}, \varepsilon_{2,3}$, and $\varepsilon_{2,4}$ are defined in table I.

The average power link between BS and the UAV-relay is $\left(\tilde{\gamma}_{2,1}, \tilde{\gamma}_{2,2}\right)$, and between the UAV-relay and $\mathcal{O}$ is $\left(\tilde{\gamma}_{2,3}, \tilde{\gamma}_{2,4}\right)$, where $\left(\tilde{\gamma}_{1,1}=\tilde{\gamma}_{2,1}=\tilde{\gamma}_{2,2}\right)$ and $\left(\tilde{\gamma}_{2,3}=\tilde{\gamma}_{2,4}\right)$.

Proof. The outage events of the outdoor user, $\mathcal{O}$, happen on different occasions. First, when the UAV-relay decodes the signal $x_{1}$ or $x_{2}$ in the first time slot incorrectly. Second, when $\mathcal{O}$ fails to detect $x_{1}$ or $x_{2}$ after the SIC in the second time slot. In other words, the outage probability of $\mathcal{O}$ mainly relays on successful decoding of signal $x_{1}$ and $x_{2}$ over both links from BS to the UAV and from the UAV to $\mathcal{O}$ after applying the SIC process, which can be expressed as

$$
\begin{gathered}
\mathrm{P}^{\mathcal{O}}=1-\underbrace{\mathcal{P}_{\mathrm{r}}\left(\gamma_{r}^{x_{1}}>\gamma_{t h_{1}}\right)}_{E_{21}} \underbrace{\mathcal{P}_{\mathrm{r}}\left(\gamma_{r}^{x_{2}}>\gamma_{t h_{2}}\right)}_{E_{22}} \\
\underbrace{\mathcal{P}_{\mathrm{r}}\left(\gamma_{\mathcal{O}}^{x_{1} \rightarrow x_{2}}>\gamma_{t h_{1}}\right)}_{E_{23}} \underbrace{\mathcal{P}_{\mathrm{r}}\left(\gamma_{\mathcal{O}}^{x_{2}}>\gamma_{t h_{2}}\right)}_{E_{24}}
\end{gathered}
$$

where $\gamma_{t h_{2}}=2^{2 R_{\mathcal{O}}}-1$, and $R_{\mathcal{O}}$ is the target data rate of $\mathcal{O}$. Hence, $E_{11}=E_{21}$. In order to omit the redundancy, the remaining events can be evaluated in a similar fashion of the aforementioned derivation from (28)-(32) with respect to their SINR values. Hence, the proof is complete.
TABLE I

THRESHOLDS OF $\mathcal{I}$ 's OP

\begin{tabular}{||c||c||c||c||}
\hline $\boldsymbol{\varepsilon}_{\mathbf{2}, \boldsymbol{n}}$ & Definition & $\boldsymbol{\varepsilon}_{\mathbf{2}, \boldsymbol{n}}$ & Definition \\
\hline$\varepsilon_{2,1}$ & $\varepsilon_{1,1}$ & $\varepsilon_{2,2}$ & $\frac{\gamma_{t h_{2}}}{\left(\alpha_{2}-\eta \alpha_{1} \gamma_{t h_{1}}\right)}$ \\
\hline$\varepsilon_{2,3}$ & $\varepsilon_{1,1}$ & $\varepsilon_{2,4}$ & $\varepsilon_{2,2}$ \\
\hline
\end{tabular}

\section{Ergodic Capacity Analysis}

It is known that EC can be computed by averaging the PDF of a fading channel over the AWGN capacity, given in [29] as

$$
\bar{C}_{i}=\mathrm{B} \times \int_{0}^{\infty} \log _{2}\left(1+\gamma_{i}\right) f_{\gamma_{i}}\left(\gamma_{i}\right) d \gamma_{i}
$$

where $i \in\{\mathcal{I}, \mathcal{O}\}$. Due to the fact that the overall capacity of a DF-relay based system is described by the weakest link capacity, EC at $\mathcal{I}$ or $\mathcal{O}$ can be determined by the minimum capacity over the total links, i.e. the link from the BS to the UAV-relay and the link between the UAV-relay to the user's destination, [30]. Therefore, we assume the link between the UAV-relay to the user's destination is the weakest link. Hence, we are interested in calculating EC at each user's node.

Proposition 3. The average channel capacity of $\mathcal{I}$ is given in (36) at the top of the next page.

Proof. By substituting (21) into (35) gives,

$$
\bar{C}_{I}=\mathrm{B} \times \int_{0}^{\infty} \log _{2}\left(1+\frac{\alpha_{1} \gamma_{\mathcal{I}}}{\alpha_{2} \gamma_{\mathcal{I}}+1}\right) f_{\gamma_{\mathcal{I}}}\left(\gamma_{\mathcal{I}}\right) d \gamma_{\mathcal{I}} .
$$

With the aid of the identity $\log _{e}(x)=\ln (x) / \ln (e)$ and some algebraic manipulations, (38) can be split into parts as

$$
\begin{aligned}
\bar{C}_{I} & =\frac{\mathrm{B}}{\ln (2)}[\underbrace{\int_{0}^{\infty} \ln \left(1+\gamma_{\mathcal{I}}\right) f_{\gamma_{\mathcal{I}}}\left(\gamma_{\mathcal{I}}\right) d \gamma_{\mathcal{I}}}_{\mathrm{A}_{1}} \\
& -\underbrace{\int_{0}^{\infty} \ln \left(1+\alpha_{2} \gamma_{\mathcal{I}}\right) f_{\gamma_{\mathrm{I}}}\left(\gamma_{\mathcal{I}}\right) d \gamma_{\mathcal{I}}}_{\mathrm{A}_{2}}] .
\end{aligned}
$$

By substituting (7) into the first integral of (39), and applying further manipulations, the following is produced,

$$
\begin{aligned}
\mathrm{A}_{1} & =\frac{1}{e^{(\mu \kappa)}} \sum_{n=0}^{\infty} \frac{\mu^{2 n+\mu} \kappa^{n}(1+\kappa)^{n+\mu}}{n ! \Gamma(n+\mu) \tilde{\gamma}_{\mathcal{I}}^{(n+\mu)}} \\
& \times \int_{0}^{\infty} \gamma_{\mathcal{I}}^{(n+\mu-1)} \exp \left(-\frac{\gamma_{\mathcal{I}} \mu(1+\kappa)}{\tilde{\gamma}_{\mathcal{I}}}\right) \ln \left(1+\gamma_{\mathcal{I}}\right) d \gamma_{\mathcal{I}} .
\end{aligned}
$$

In order to solve the above integral, we need to transform the exponential and logarithmic functions into their corresponding Meijer G-function representations defined in [31, 8.4.3.1, 8.4.6.5]. The equivalent form of Meijer G-function is applied 


$$
\begin{aligned}
\bar{C}_{\mathcal{I}} & =\frac{\mathrm{B}}{\ln (2) e^{(\mu \kappa)}} \sum_{n=0}^{\infty} \frac{\mu^{2 n+\mu} \kappa^{n}(1+\kappa)^{n+\mu}}{n ! \Gamma(n+\mu) \tilde{\gamma}_{\mathcal{I}}^{(n+\mu)}} \times\left(\mathrm{H}_{2,3}^{3,1}\left[\frac{\mu(1+\kappa)}{\tilde{\gamma}_{\mathcal{I}}} \mid \begin{array}{l}
(-(n+\mu), 1),(1-(n+\mu), 1) \\
0,(-(n+\mu), 1),(-(n+\mu), 1)
\end{array}\right]\right. \\
& -\left(\alpha_{2}\right)^{-(n+\mu)} \times \mathrm{H}_{2,3}^{3,1}\left[\begin{array}{ll}
\left.\left.\frac{\mu(1+\kappa)}{\alpha_{2} \tilde{\gamma}_{\mathcal{I}}} \mid \begin{array}{l}
(-(n+\mu), 1),(1-(n+\mu), 1) \\
0,(-(n+\mu), 1),(-(n+\mu), 1)
\end{array}\right]\right)
\end{array}\right.
\end{aligned}
$$

$$
\begin{aligned}
\bar{C}_{\mathcal{O}} & =\frac{\mathrm{B}}{\ln (2) e^{(\mu \kappa)}} \sum_{n=0}^{\infty} \frac{\mu^{2 n+\mu} \kappa^{n}(1+\kappa)^{n+\mu}}{n ! \Gamma(n+\mu) \tilde{\gamma}_{\mathcal{O}}^{(n+\mu)}} \\
& \times\left(\left(\eta \alpha_{1}+\alpha_{2}\right)^{-(n+\mu)} \times \mathrm{H}_{2,3}^{3,1}\left[\frac{\mu(1+\kappa)}{\left(\eta \alpha_{1}+\alpha_{2}\right) \tilde{\gamma}_{\mathcal{O}}} \mid \begin{array}{l}
(-(n+\mu), 1),(1-(n+\mu), 1) \\
0,(-(n+\mu), 1),(-(n+\mu), 1)
\end{array}\right]\right. \\
& \left.-\left(\eta \alpha_{1}\right)^{-(n+\mu)} \times \mathrm{H}_{2,3}^{3,1}\left[\frac{\mu(1+\kappa)}{\eta \alpha_{1} \tilde{\gamma}_{\mathcal{O}}} \mid \begin{array}{l}
(-(n+\mu), 1),(1-(n+\mu), 1) \\
0,(-(n+\mu), 1),(-(n+\mu), 1)
\end{array}\right]\right)
\end{aligned}
$$

by calling the Fox H-function given in [32, 6.2.8]. Thus,

$$
\begin{aligned}
\mathrm{A}_{1} & =\frac{1}{e^{(\mu \kappa)}} \sum_{n=0}^{\infty} \frac{\mu^{2 n+\mu} \kappa^{n}(1+\kappa)^{n+\mu}}{n ! \Gamma(n+\mu) \tilde{\gamma}_{I}^{(n+\mu)}} \\
& \times \int_{0}^{\infty} \gamma_{\mathcal{I}}^{(n+\mu-1)} \mathrm{H}_{0,1}^{1,0}\left[\frac{\gamma_{\mathcal{I}} \mu(1+\kappa)}{\tilde{\gamma}_{\mathcal{I}}} \mid \begin{array}{c}
- \\
(0,1)
\end{array}\right] \\
& \times \mathrm{H}_{2,2}^{1,2}\left[\gamma_{\mathcal{I}} \mid \begin{array}{l}
(1,1),(1,1) \\
(1,1),(0,1)
\end{array}\right] d \gamma_{\mathcal{I} .}
\end{aligned}
$$

Applying the identity in [33] gives,

$$
\begin{aligned}
\mathrm{A}_{1} & =\frac{1}{e^{(\mu \kappa)}} \sum_{n=0}^{\infty} \frac{\mu^{2 n+\mu} \kappa^{n}(1+\kappa)^{n+\mu}}{n ! \Gamma(n+\mu) \tilde{\gamma}_{\mathcal{I}}^{(n+\mu)}} \\
& \times \mathrm{H}_{2,3}^{3,1}\left[\begin{array}{l|l}
\frac{\mu(1+\kappa)}{\tilde{\gamma}_{\mathcal{I}}} & (-(n+\mu), 1),(1-(n+\mu), 1) \\
0,(-(n+\mu), 1),(-(n+\mu), 1)
\end{array}\right] .
\end{aligned}
$$

Similarly, the second integral of (39) is evaluated by following the above steps, which gives the following expression.

$$
\begin{aligned}
\mathrm{A}_{2} & =\frac{1}{e^{(\mu \kappa)}} \sum_{n=0}^{\infty} \frac{\mu^{2 n+\mu} \kappa^{n}(1+\kappa)^{n+\mu}}{n ! \Gamma(n+\mu) \tilde{\gamma}_{\mathcal{I}}^{(n+\mu)}} \times\left(\alpha_{2}\right)^{-(n+\mu)} \\
& \times \mathrm{H}_{2,3}^{3,1}\left[\frac{\mu(1+\kappa)}{\alpha_{2} \tilde{\gamma}_{\mathcal{I}}} \mid \begin{array}{l}
(-(n+\mu), 1),(1-(n+\mu), 1) \\
0,(-(n+\mu), 1),(-(n+\mu), 1)
\end{array}\right] .
\end{aligned}
$$

Ultimately, we substitute (42) and (43) in (39), which produces the final expression of EC of $\mathcal{I}$ as stated in Prop. 3 , and the proof is complete.

Proposition 4. The average channel capacity of the $\mathcal{O}$ NOMA user is given in (37) at the top of this page.

\section{E. Throughput Analysis}

The system throughput of each user in a delay-limited transmission mode can be evaluated by its outage probability or EC. Therefore, the corresponding outage throughput of a two-user NOMA system is given as in [34] as follows,

$$
\mathrm{T}_{i}^{\mathrm{OP}}=\left(1-\mathrm{P}^{\mathrm{i}}\right) \times R_{i}
$$

where $i \in\{\mathcal{I}, \mathcal{O}\}, R$ is the user's data target rate. Therefore, we substitute (27) and (33) in (44) to obtain the throughput of $\mathcal{I}$ and $\mathcal{O}$, respectively.

\section{F. Energy Efficiency (EE) Analysis}

The EE for a delay-limited system can be defined as the ratio of EC and the total power consumption of the system. Therefore, EE can be given as in [35]

$$
E E=\frac{R_{\text {total }}}{\mathrm{P}_{\text {total }}}=\frac{\tilde{C}_{\mathcal{I}}+\tilde{C}_{\mathcal{O}}}{\delta\left(\alpha_{1} \mathcal{P}_{\mathrm{r}}+\alpha_{2} \mathcal{P}_{\mathrm{r}}\right)+\mathrm{P}_{c}-E_{\text {total }}}
$$

where $\delta$ indicates the energy amplifier efficiency, $\tilde{C}_{\mathcal{I}}$ and $\tilde{C}_{\mathcal{O}}$ are given in (36) and (37), respectively. $\mathcal{P}_{\mathrm{c}}$ is known as the circuit power, and $E_{\text {total }}$ is the overall harvested power of the considered system, which can be expressed as

$$
E_{\text {total }}=(1-\tau) \delta \sum^{i}\left(\left|h_{i}\right|^{2} \sum_{j=1}^{2} \alpha_{j} \mathcal{P}_{\mathrm{r}}\right)
$$

where $i \in\{\mathcal{I}, \mathcal{O}\}$ and $j \in\{1,2\}$.

\section{Numerical Results}

The parameters used in this section are presented in table II. The channel coefficients have $\kappa-\mu$ distribution, which are generated as described in [36]. The channel is considered to be quasi-static where it remains fixed during one symbol duration, but changes randomly over consecutive symbol intervals. In each simulation run we generate $10^{6}$ symbols.

Fig. 2 shows the OP of $\mathcal{I}$ over different channel fading parameters as a function of $\mathcal{P}_{\mathrm{s}}$. In Fig. 2a, the parameter $\mu$ is changed for a wide range of values, while $\kappa=0$. As can be noted from the figure, the OP of $\mathcal{I}$ improves slowly with $\mathcal{P}_{\mathrm{s}}$ for the case of $\mu=1$. Such performance is due to the severe attenuation caused by the building walls and large 
TABLE II

SIMULATION PARAMETERS.

\begin{tabular}{cccc}
\hline Parameter & Value & Parameter & Value \\
\hline Transmit power $\left(\mathcal{P}_{\mathrm{s}}\right)$ & $(0-40) \mathrm{dBm}$ & Coordinates of the BS $\left(x_{s}, y_{s}, z_{s}\right)$ & $(0,0,35) \mathrm{m}$ \\
Carrier Frequency $(f)$ & $1200 \mathrm{MHz}$ & Coordinates of the UAV-relay $\left(x_{r}, y_{r}, z_{r}\right)$ & $(400,20,80) \mathrm{m}$ \\
System bandwidth $(\mathrm{B})$ & $1 \mathrm{MHz}$ & Coordinates of the building $\left(x_{b}, y_{b}, z_{b}\right)$ & $(1500,98,35) \mathrm{m}$ \\
Noise power $(\sigma)$ & $-174 \mathrm{dBm}$ & Coordinates of $\mathcal{I}\left(x_{\mathcal{I}}, y_{\mathcal{I}}, z_{\mathcal{I}}\right)$ & $(1502,100,3) \mathrm{m}$ \\
Target data rates $\left(R_{\mathcal{I}}, R_{\mathcal{O}}\right)$ & $(0.5,1.5) \mathrm{b} / \mathrm{s} / \mathrm{Hz}$ & Coordinates of $\mathcal{O}\left(x_{\mathcal{O}}, y_{\mathcal{O}}, z_{\mathcal{O}}\right)$ & $(1000,50,3) \mathrm{m}$ \\
Power allocation factor of $\mathcal{I}\left(\alpha_{1}\right)$ & 0.9 & Indoor distance of $\mathcal{I}\left(d_{i n}\right)$ & $2 \mathrm{~m}$ \\
SIC imperfect factor $(\eta)$ & $3.5 \times 10^{-2}, 3.5 \times 10^{-3}$ & Perpendicular loss $\left(L_{p c}\right)$ & $7 \mathrm{~dB}$ \\
Circuit power $\left(\mathcal{P}_{\mathrm{c}}\right)$ & $5 \mathrm{Watt}$ & Loss of internal walls $\left(L_{i}\right)$ & $7 \mathrm{~dB}$ \\
Time division factor $(\tau)$ & 0.6 & Number of internal walls $(n)$ & 0 \\
Energy amplifier efficiency $(\delta)$ & $85 \%$ & Indoor path loss parameter $(\chi)$ & $0.6 \mathrm{~dB} / \mathrm{m}$ \\
Parallel penetration loss $\left(L_{p t}\right)$ & $20 \mathrm{~dB}$ & & \\
\hline
\end{tabular}
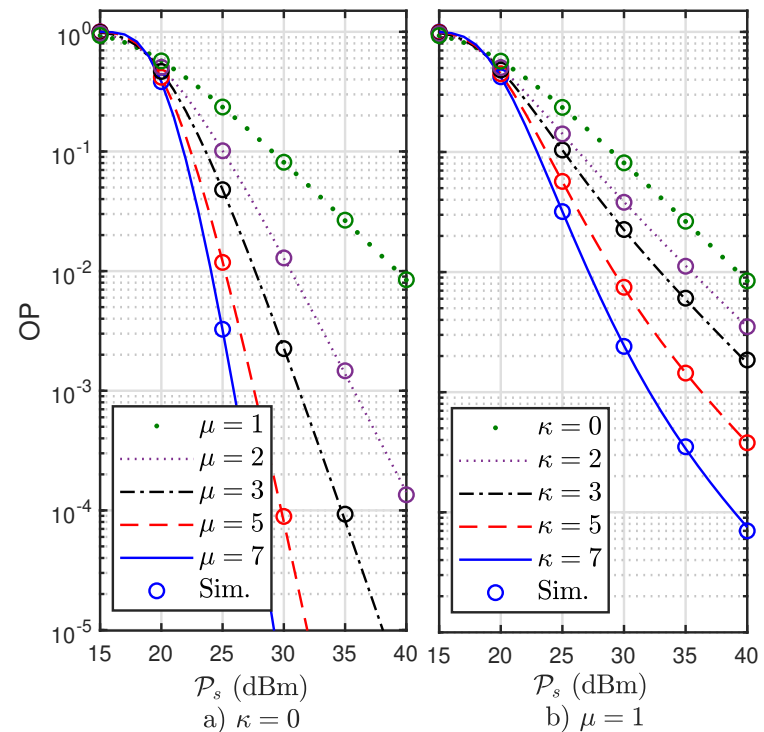

Fig. 2. Outage probability of $\mathcal{I}$ in different fading conditions versus $\mathcal{P}_{\mathrm{s}}$.

distance path-loss. For $\mu=5$, OP improves significantly with $\mathcal{P}_{\mathrm{S}}$ because the channel gain is generally high for such values of $\mu$. Similarly, Fig. $2 b$ leads to the same conclusion, but with less enhancements in OP. To illustrate, manipulating the fading parameter $\mu$ attains more effective results. Hence, other parameters such as indoor distance, number of indoor walls, and materials of the walls play a major role in obtaining high OP. In general, $\mathcal{I}$ can experience various channel fading values for $\kappa$ and $\mu$. Nevertheless, it is generally considered as NLOS.

Fig. 3 shows the OP of $\mathcal{O}$ over various $\kappa$ and $\mu$ values. Unlike the case of $\mathcal{I}$ in Fig. 2, this figure shows that the OP of $\mathcal{O}$ outperforms the OP of $\mathcal{I}$ over all ranges of channel fading components. This is because of the lesser path-loss and closer distance to the transmitter. It also shows the difference between the outdoor and indoor environment, where the challenges are more severe for the indoor conditions. It is also found that the channel fading parameter $\mu$ has more effect on OP than $\kappa$ component. For example, when $\kappa=0$ and $\mu=7$, the OP
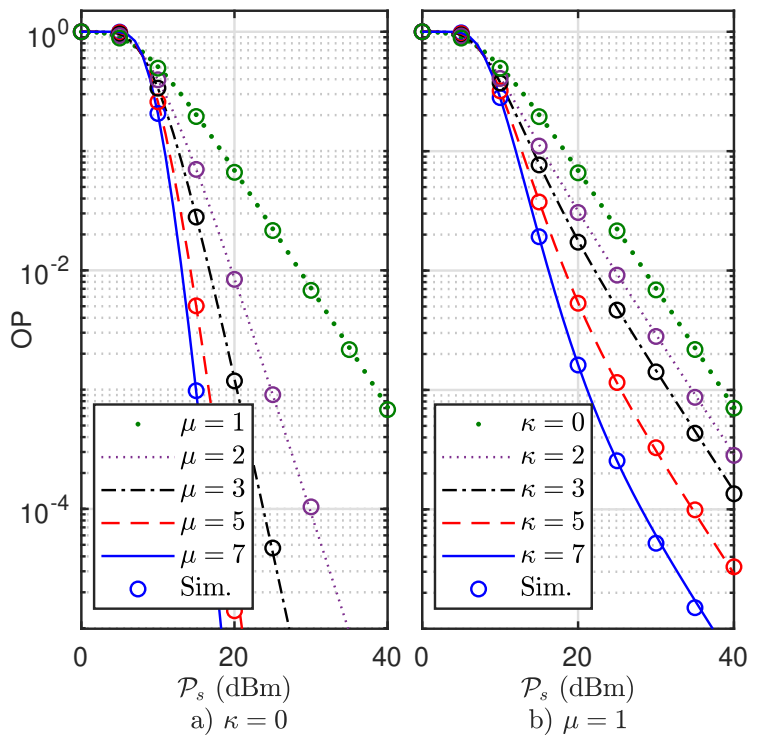

Fig. 3. Outage probability of $\mathcal{O}$ in different fading conditions versus $\mathcal{P}_{\mathrm{s}}$

of $10^{-4}$ can be easily attained using less than $20 \mathrm{dBm}$ of transmitting power. However, when $\mu=1$ and $\kappa=7$, more than $20 \mathrm{dBm}$ of transmitting power is needed to reach the same $\mathrm{OP}$ value. On the other hand, $\mathcal{I}$ requires about $10 \mathrm{dBm}$ to achieve $10^{-4}$ of OP under the same channel conditions.

Fig. 4 shows the impact of the residual interference caused by $\mathcal{I}$ with the case of imperfect SIC scenario. It only presents the OP of $\mathcal{O}$ since $\mathcal{I}$ does not apply SIC. Therefore, a couple of different residual interference values is tested in that figure to show the significant impact of $\eta$. To illustrate, it can be observed that a noticeable difference of about $4 \mathrm{dBm}$ is obtained when $\eta$ changes from 0.0035 to become 0.035 . This amount of remaining noise can significantly degrade the OP performance of $\mathcal{O}$. In addition to that, the channel fading parameters play a major role in the total performance, where an increase in $\mu$ can lead to better performance.

In contrast, Fig. 5 illustrates the impact of the power allocation factor $\left(\alpha_{1}\right)$ within different transmitting power values. 


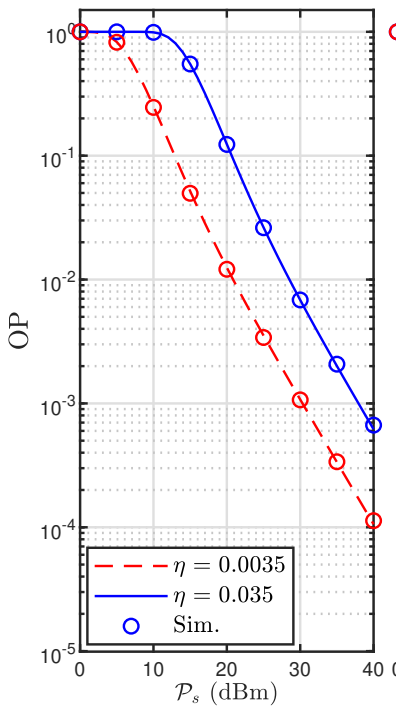

a) $\kappa=3, \mu=1$

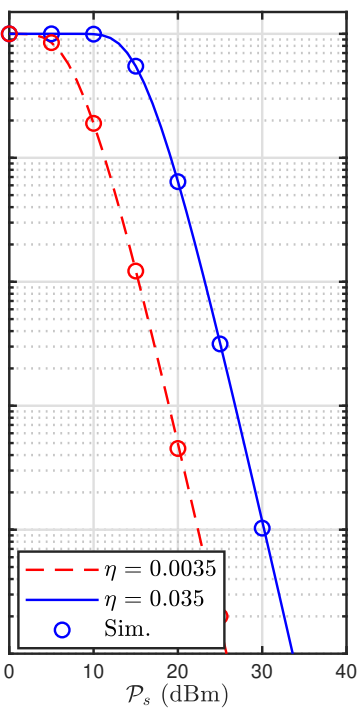

b) $\kappa=0, \mu=3$
Fig. 4. Outage probability of $\mathcal{O}$ for different values of $\eta$ versus $\mathcal{P}_{\mathrm{s}}$.
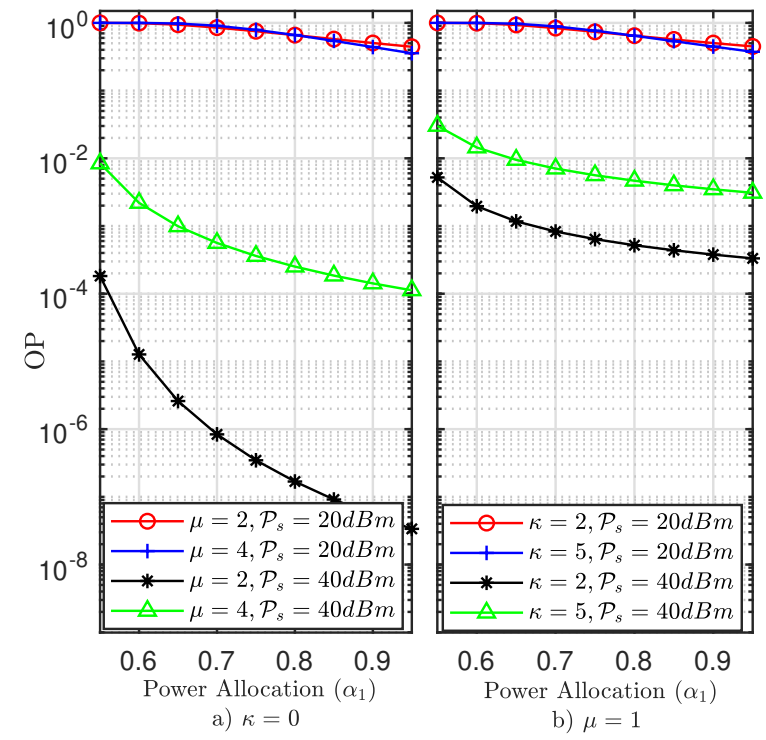

Fig. 5. Outage probability of $\mathcal{I}$ versus $\alpha_{1}$ with $\eta=0$.

The optimal choice of $\alpha_{1}$ can be determined at the overlapping points between both users. For example, at low transmitting power such as $20 \mathrm{dBm}, \alpha=0.85$ would be the best selection, and so forth. In general, $\mathcal{I}$ attains more improvements in terms of OP when $\alpha_{1}$ increases. In addition, the fading channel parameters have a direct impact on the system performance. As it can be seen from that figure, when $\mu$ varies the performance becomes better with large transmitting power. Furthermore, at low transmitting power, there is no significant difference between the two scenarios of the fading channel assignments. However, a remarkable change appears between the two cases at high transmitting powers .

Similarly, Fig. 6 shows the impact of power allocation

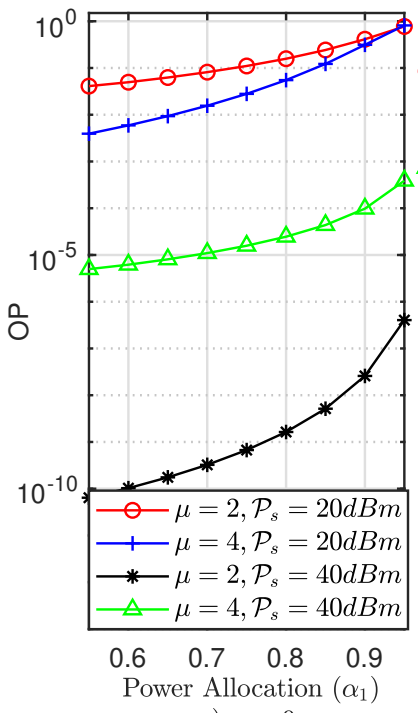

a) $\kappa=0$

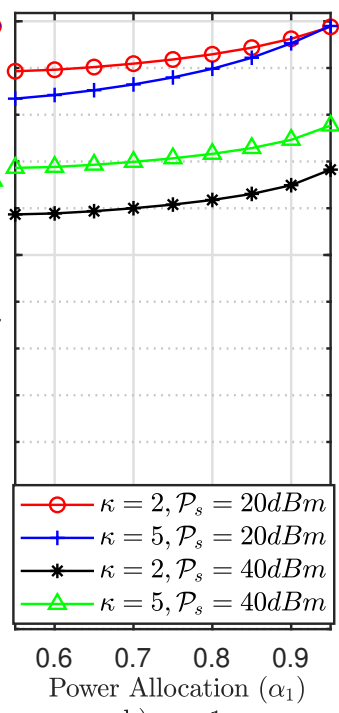

b) $\mu=1$
Fig. 6. Outage probability of $\mathcal{O}$ versus power allocation factor with $\eta=0$.

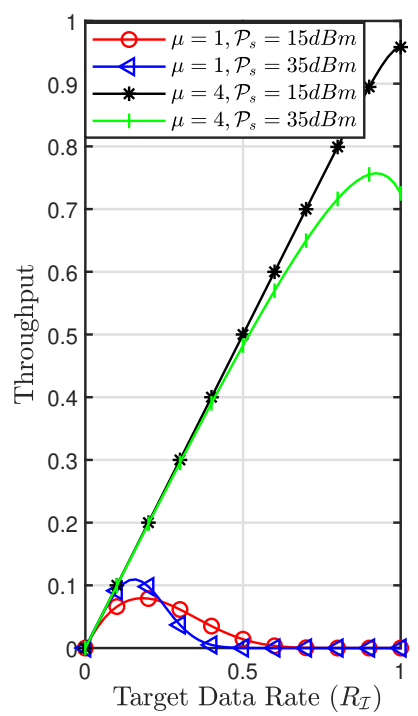

a) $\kappa=0$

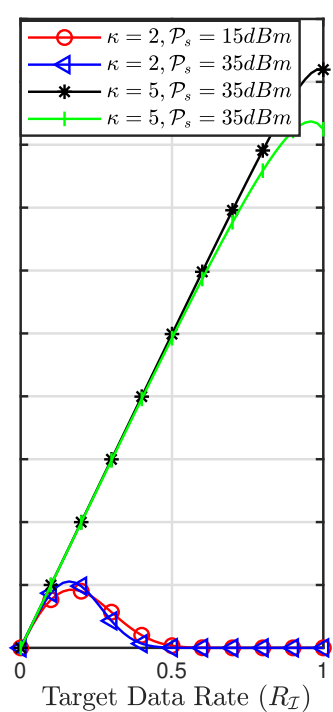

a) $\kappa=0$
Fig. 7. Throughput of $\mathcal{I}$ in different $\mathcal{P}_{\mathrm{s}}$ versus target data rates.

assignment on the OP of $\mathcal{O}$. It indicates that increasing the amount of $\alpha_{1}$ leads to more outage for $\mathcal{O}$. Therefore, power allocation must be carefully distributed between the NOMA users to distinguish their signals at the receiver side. A similar approach of manipulating the fading channels' parameters is reapplied and the results reveal that the $\mu$ fading parameter has a major impact on the system performance. Both of the last figures can be used to identify the optimal power selection for both users by determining the overlapping points over different values of transmitting power.

Fig. 7 shows the throughput performance of $\mathcal{I}$ corresponding to its predefined data rate over some particular values of transmitting power. We assume that high QoS is aimed for $\mathcal{O}$, 


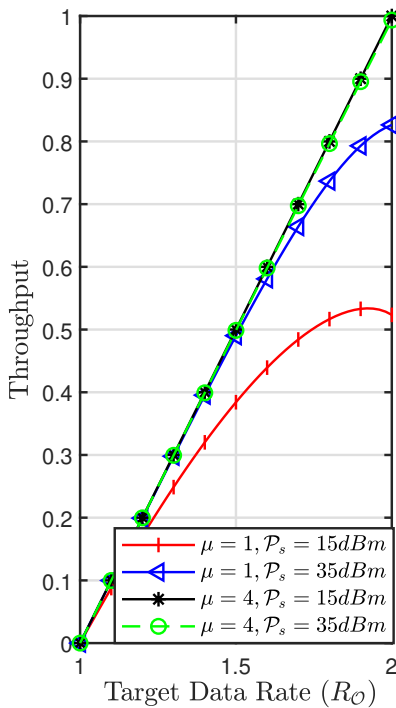

a) $\kappa=0$

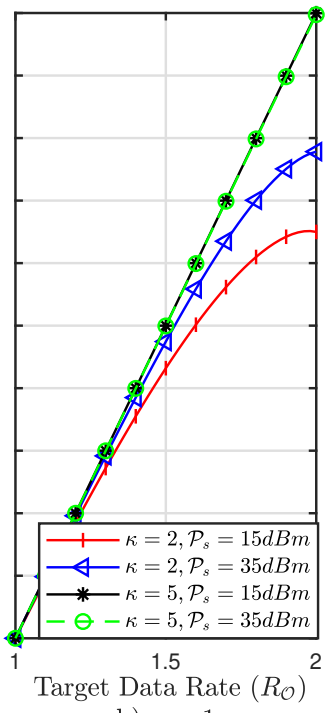

b) $\mu=1$
Fig. 8. Throughput of $\mathcal{O}$ for different $\mathcal{P}_{\mathrm{s}}$ versus target data rates with $\eta=0$.

so its target data rate is always higher than $\mathcal{I}$. Therefore, the results are attained based on a particular range of target data rates as shown in Fig. 7. It is observed that higher throughput is attained at high target data rates for $\mathcal{I}$. It also shows that different values of fading parameters can significantly affect the system performance. Manipulating $\mu$ provides more effective results than the $\kappa$ parameter.

Unlike Fig. 7, the throughput performance of $\mathcal{O}$ can be seen in Fig. 8, where the results show some potentials over $\mathcal{I}$. It is because of the privileges assigned to $\mathcal{O}$ such as high QoS. At high channel fading parameters, the throughput performance is almost similar. However, at low fading parameters and transmitting power a distinguished performance can be observed. In general, $\mathcal{O}$ outperforms $\mathcal{I}$ in terms of throughput analysis because of different factors such as power allocation, channel gain, and environment conditions.

As can be noted from Fig. 9, the $\mathcal{I}$ performance is significantly affected by the time splitting factor $\tau$ where various levels of throughput are presented. The results indicate that in order to maximize the throughput, the time division factor, specified for the harvested energy amount to conveying the NOMA message to the final destinations in the second time slot, should be more than 0.5 at low transmitting power. Similarly, at high transmitting power, more effective results can be obtained by considering $\tau$ at more than 0.2 . These results can be changed accordingly based on multiple factors such as power components, channel characteristics, path loss exponent and other factors including $(\tau)$. Hence, increasing $\mu$ has more impact than $\kappa$, as can be seen from that figure.

In contrast to Fig. 9, the results in Fig. 10 demonstrate throughput analysis versus the time division factor $\tau$ for $\mathcal{O}$. It can be seen that $\mathcal{O}$ gradually achieves high throughput over a low transmit power of $15 \mathrm{dBm}$ with $\tau=0.3$ than $\mathcal{I}$, which requires $\tau=0.5$ to gain some throughput. Nevertheless, $\mathcal{O}$ exceeds the limit of $\mathcal{I}$ throughout in many different occasions

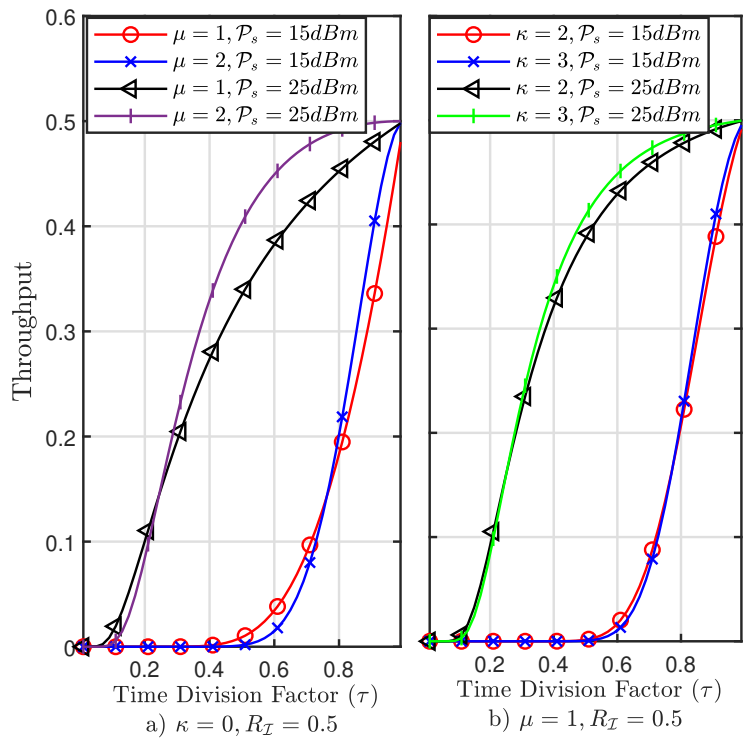

Fig. 9. Throughput of $\mathcal{I}$ in different $\mathcal{P}_{\mathrm{s}}$ versus time splitting factor $(\tau)$.

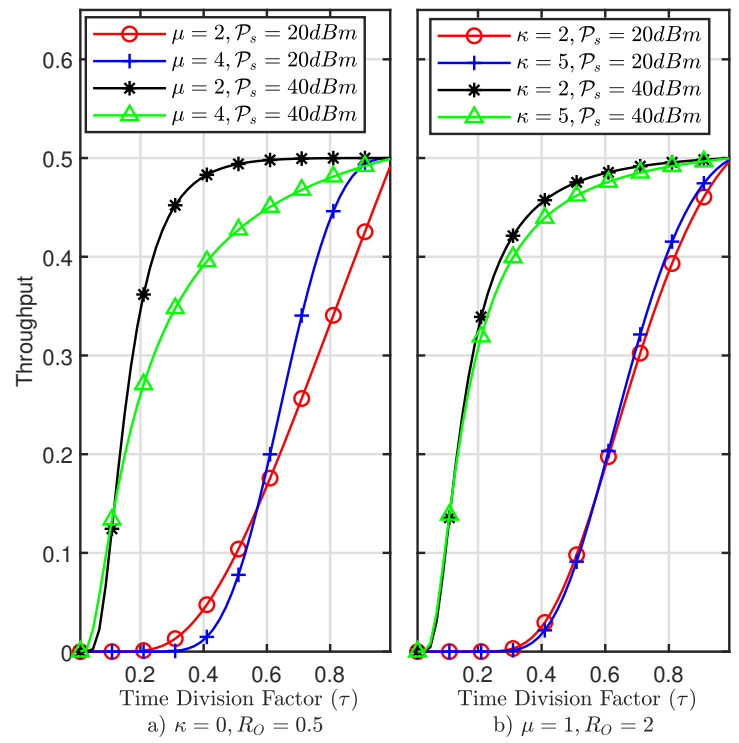

Fig. 10. Throughput of $\mathcal{I}$ in different $\mathcal{P}_{\mathrm{s}}$ versus time splitting factor.

over multiple transmit power and channel fading parameters. For instance, at $15 \mathrm{dBm}, \mathcal{O}$ requires $\tau=0.6$ to accomplish about $0.2 \mathrm{bits} / \mathrm{sec}$. while $\mathcal{I}$ needs about $\tau=0.8$ under similar transmit power. It also indicates that $\mathcal{O}$ outperforms $\mathcal{I}$ for all cases of channel fading assignments.

Fig. 11 shows another crucial performance metric known as the average channel capacity. Specifically, it plots the individual rates of both users versus the total transmit power. It can be observed from Fig. 11 that $\mathrm{EC}$ of $\mathcal{O}$ is manipulated by the impact factor of the residual interference. It becomes worse than the ideal case when $\eta$ aims to be higher, but it still performs better than $\mathcal{I}$ at all range of transmit power. However, $\mathcal{I}$ achieves about $2 \times 10^{6}-3 \times 10^{6}(\mathrm{~b} / \mathrm{s} / \mathrm{Hz})$ over 28 


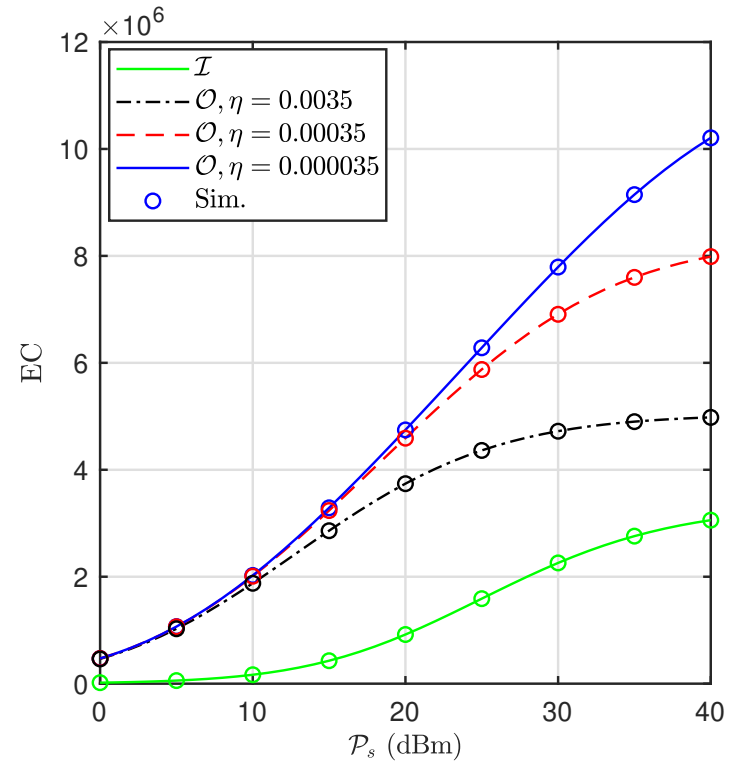

Fig. 11. Ergodic capacity of both users versus $\mathcal{P}_{\mathrm{s}}$ over different vales of $\eta$, and $\kappa=0, \mu=1$ for all channels.

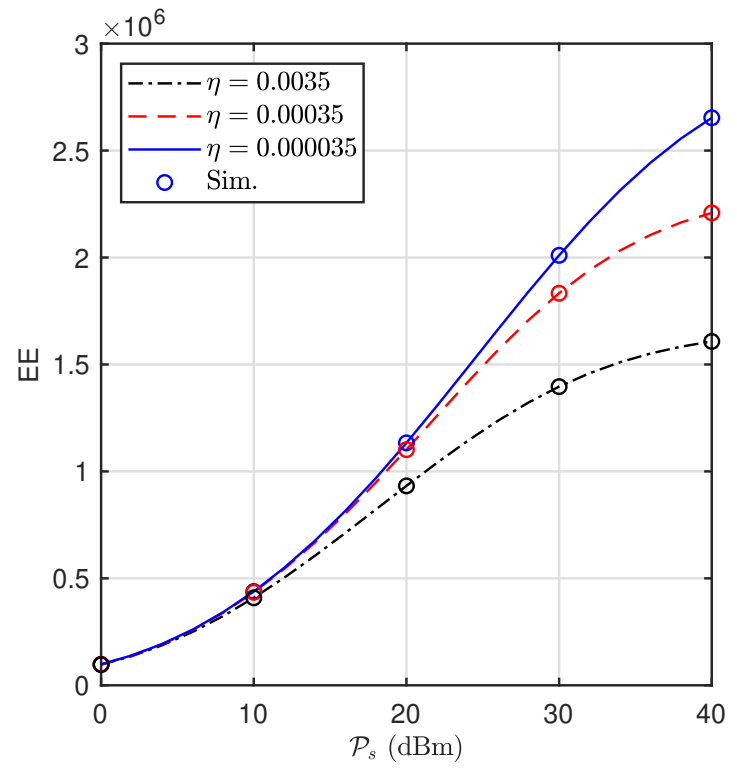

Fig. 12. Energy efficiency versus $\mathcal{P}_{\mathrm{s}}$ with $\kappa=0, \mu=1$.

$\mathrm{dBm}$ while $\mathcal{O}$ gains between $7 \times 10^{6}-12 \times 10^{6}(\mathrm{~b} / \mathrm{s} / \mathrm{Hz})$ at the absence of residual interference, i.e., $\eta=0$. Ultimately, the residual interference, path-loss gain, fading parameters have a significant influence on the overall system performance.

Fig. 12 studies the overall system energy efficiency as a function of the transmit power for different conditions of SIC. It can be seen from Fig. 12 that EE increases as the transmit power increases, but it gradually decreases at very large transmit power values due to the imperfect SIC phenomenon. In the best scenario of $\eta$, EE linearly increases over the whole range of transmit power. However, when $\eta$ intends to increased, EE exhibits almost floor performance at high transmit power. Therefore, different system parameters can play a major role in enhancing the overall system performance.

\section{CONCLUSion ANd Future Work}

In this paper, a performance analysis is performed for a mix of indoor and outdoor NOMA users, where both users can be served via an outdoor-to-indoor and outdoor-to-outdoor channels. Different practical challenges are considered including imperfect SIC and various fading conditions. The channel gains are sorted based on the product of the instantaneous channels' gains with their distance-dependant path loss, where $\mathcal{I}$ has different channel attributes than $\mathcal{O}$. We studied the path-loss model of $\mathcal{I}$ in generalized $\kappa-\mu$ fading distribution to better describe the channel gain when both scenarios of LOS and NLOS are present. Furthermore, we evaluated a variety of vital performance metrics such as OP and EC, as well as throughput and energy efficiency, and provided exact analytical expressions. The obtained results provide an insight into the impact of different key factors, such as power allocation, time splitting, $\kappa$ and $\mu$ fading, and residual interference, on the total system performance. It is shown that the performance of $\mathcal{I}$ degrades more than $\mathcal{O}$ due to its environment defined by its distinct channel attributions. The derived analytical expressions are validated by extensive simulations which confirmed the accuracy of our analysis.

Future work will explore various indoor-outdoor multipleinput-multiple-output (MIMO) NOMA system scenarios.

\section{LIST OF ACRONYMS}

NOMA non-orthogonal multiple accesses.

SWIPT simultaneous wireless information and power transfer.

C-NOMA cooperative non-orthogonal multiple accesses.

UAV unmanned aerial vehicle.

DF decode-and-forward.

OP outage probability.

EC ergodic capacity.

EE energy efficiency.

SIC successive interference cancellation.

QoS quality of service.

5G fifth generation.

SC superposition coding.

OMA orthogonal multiple access.

CSI channel state information.

HD half-duplex.

FR fixed relaying.

ISDF incremental-selective DF.

AF amplify-and-forward.

SNR signal-to-noise ratio.

VLC visible light communication.

F-NOMA fixed NOMA.

CR-NOMA cognitive radio NOMA.

RNRF random near user and random far user.

NNNF nearest near user and nearest far user.

NNFF nearest near user and farthest far user.

SUAAs sub-carrier user assignment algorithms. 


$\begin{array}{ll}\text { BS } & \text { base station. } \\ \text { SINR } & \text { signal to interference plus noise ratio. } \\ \text { TSR } & \text { time switching-based relaying. } \\ \text { LOS } & \text { line of sight. } \\ \text { NLOS } & \text { non-line of sight. } \\ \text { PDF } & \text { probability density function. } \\ \text { CDF } & \text { cumulative density function. } \\ \text { AWGN } & \text { additive white Gaussian noise. } \\ \text { EH } & \text { energy harvesting. } \\ \text { IP } & \text { information process. } \\ \text { ML } & \text { maximum likelihood. } \\ \text { MIMO } & \text { multiple-input-multiple-output. } \\ \text { SF-CDRT } & \text { selective DF with coordinated direct and relay } \\ \text { BER } & \text { transmission. } \\ & \text { bit error rate. }\end{array}$

\section{LIST OF SYMBOLS}

$\kappa \quad$ Channel fading parameter.

$\mu \quad$ Channel fading parameter.

$\eta \quad$ SIC Imperfect factor.

$\mathcal{I}$ Indoor user.

$\mathcal{O}$ Outdoor user.

$r \quad$ UAV-relay.

$g_{i} \quad$ Small scale fading.

$d_{s r} \quad$ Distance between BS and UAV-relay.

$f \quad$ Carrier frequency.

$L_{f s} \quad$ Free space path loss.

$d_{\text {in }}$ Distance between interior wall and indoor user.

$d_{r \mathcal{I}} \quad$ Distance between UAV-relay and external wall.

$L_{t} \quad$ Transition propagation path loss.

$L_{p c} \quad$ Perpendicular path loss.

$L_{p t} \quad$ Parallel penetration loss.

$\theta \quad$ Grazing angle between UAV-relay and exterior wall.

$L_{i n} \quad$ Indoor propagation path loss.

$n \quad$ Number of internal walls.

$L_{i} \quad$ Internal wall lose.

$\chi \quad$ Indoor path loss parameter.

$h \quad$ Channel frequency response.

$\mathcal{P} \quad$ Signal power.

$\mathbb{E}[$.$] \quad The expectation operator.$

$\Omega \quad$ The mean power of $h$.

$\mathrm{I}_{v}($.$) Modified Bassel function.$

$\mathcal{Q}_{(.)} \quad$ Generalized Marcum- $Q$ function.

$\alpha_{1} \quad$ Power allocation factor of $\mathcal{I}$.

$\alpha_{2} \quad$ Power allocation factor of $\mathcal{O}$.

$\mathcal{P}_{\mathrm{S}} \quad$ Total transmitted power at BS.

$\tau \quad$ Time splitting factor.

$x_{1} \quad$ Symbol of $\mathcal{I}$.

$x_{2} \quad$ Symbol of $\mathcal{O}$.

$\hat{x}_{1} \quad$ Estimated symbol of $\mathcal{I}$.

$\mathbb{S} \quad$ Set of all possible constellation points.

$\hat{x}_{2} \quad$ Estimated symbol of $\mathcal{O}$.

$T$ Total time interval of the frame.

$\mathcal{P}_{\mathrm{r}} \quad$ Transmitted power at UAV-relay.

$R_{\mathcal{I}} \quad$ Indoor target data rate.

$\tilde{\gamma}_{1,1} \quad$ Average power link between BS and UAV-relay.

$\tilde{\gamma}_{1,2}$ Average power link between $\mathrm{UAV}$-relay and $\mathcal{I}$.
$R_{\mathcal{O}} \quad$ Outdoor target data rate.

$R \quad$ Data target rate.

$\delta \quad$ Energy amplifier efficiency.

$\tilde{C}_{\mathcal{I}} \quad$ Ergodic capacity of indoor user.

$\tilde{C}_{\mathcal{O}} \quad$ Ergodic capacity of outdoor user.

$\mathcal{P}_{\mathrm{c}} \quad$ Circuit power.

$E_{\text {total }}$ Total harvested power.

B System bandwidth.

$\sigma \quad$ Noise power.

$P L \quad$ Path loss.

$\rho \quad$ Signal to noise ratio.

$\gamma \quad$ Instantaneous SNR.

$\tilde{\gamma} \quad$ Average SNR.

$\alpha \quad$ Power allocation factor.

$x_{s c} \quad$ Superimposed NOMA signal.

$d_{r \mathcal{O}}$ Distance between UAV-relay and outdoor user.

$d_{r \mathcal{I}} \quad$ Distance between UAV-relay and indoor user.

$d_{\text {out }}$ Distance between UAV-relay and exterior wall.

$f_{\gamma(\gamma)}$ Probability density function.

$F_{\gamma(\gamma)}$ Cumulative density function.

$\hat{x} \quad$ Estimated symbol.

$\tilde{\gamma}_{2,3} \quad$ Average power link between UAV-relay and $\mathcal{O}$.

$\mathrm{P}^{\mathcal{O}}$ Outage Propability of $\mathcal{O}$.

\section{REFERENCES}

[1] A. S. Alqahtani and E. Alsusa, "Performance analysis of downlink NOMA system over $\alpha-\eta-\mu$ generalized fading channel," in Proc. IEEE Veh. Technol. Conf. (VTC), Jun. 2020, pp. 1-5.

[2] Q. Li, H. Niu, A. Papathanassiou, and G. Wu, "5G network capacity: Key elements and technologies," IEEE Veh. Technol. Mag., vol. 9, no. 1, pp. 71-78, Mar. 2014.

[3] M. Agiwal, A. Roy, and N. Saxena, "Next generation 5G wireless networks: A comprehensive survey," IEEE Commun. Surveys Tuts., vol. 18, no. 3, pp. 1617-1655, Jun. 2016.

[4] Z. Ding, J. C. Y. Liu, M. E. Q. Sun, C. L. I, , and H. V. Poor, "Application of non-orthogonal multiple access in LTE and 5G networks," IEEE Commun. Mag., vol. 55, no. 3, pp. 185-191, Feb. 2017.

[5] S. R. Islam, N. Avazov, O. A. Dobre, and K.-S. Kwak, "Power-domain non-orthogonal multiple access (NOMA) in 5G systems: Potentials and challenges," IEEE Commun. Surveys Tuts., vol. 19, no. 2, pp. 721-742, Feb. 2016.

[6] L. Dai, B. Wang, Z. Ding, Z. Wang, S. Chen, and L. Hanzo, "A survey of non-orthogonal multiple access for 5G," IEEE Commun. Surveys Tuts., vol. 20, no. 3, pp. 2294-2323, May 2018.

[7] Z. Ding, Z. Yang, P. Fan, and H. V. Poor, "On the performance of non-orthogonal multiple access in $5 \mathrm{G}$ systems with randomly deployed users," IEEE Signal Process. Lett., vol. 21, no. 12, pp. 1501-1505, Dec. 2014.

[8] Z. Yang, Z. Ding, P. Fan, and G. K. Karagiannidis, "On the performance of non-orthogonal multiple access systems with partial channel information," IEEE Trans. Commun., vol. 64, no. 2, pp. 654-667, Dec. 2016.

[9] Z. Ding, M. Peng, and H. V. Poor, "Cooperative non-orthogonal multiple access in 5G systems," IEEE Commun. Lett., vol. 19, no. 8, pp. 14621465, Jun. 2015.

[10] Q. Y. Liau and C. Y. Leow, "Successive user relaying in cooperative NOMA system," IEEE Wireless Commun. Lett., vol. 8, no. 3, pp. 921924, Feb. 2019.

[11] H. Liu, Z. Ding, K. J. Kim, K. S. Kwak, and H. V. Poor, "Decodeand-forward relaying for cooperative NOMA systems with direct links," IEEE Trans. Wirel. Commun., vol. 17, no. 12, pp. 8077-8093, Oct. 2018.

[12] L. Bariah, S. Muhaidat, and A. Al-Dweik, "Error probability analysis of NOMA-Based relay networks with SWIPT," IEEE Commun. Lett., vol. 23, no. 7, pp. 1223-1226, May 2019.

[13] - "Error performance of NOMA-Based cognitive radio networks with partial relay selection and interference power constraints," IEEE Trans. Commun., vol. 68, no. 2, pp. 765-777, May 2020. 
[14] M. Guangchen, "Joint beamforming and power allocation for wireless powered UAV-assisted cooperative NOMA systems," EURASIP J. Wirel. Commun. Netw., no. 55, pp. 1-14, Mar. 2020.

[15] X. Liang, Y. Wu, D. W. K. Ng, Y. Zuo, S. Jin, and H. Zhu, "Outage performance for cooperative NOMA transmission with an AF relay," IEEE Commun. Lett., vol. 21, no. 11, pp. 2428-2431, Mar. 2017.

[16] S. K. Zaidi, S. F. Hasan, and X. Gui, "Outage analysis of ground-aerial NOMA with distinct instantaneous channel gain ranking," IEEE Trans. Veh. Technol., vol. 68, no. 11, pp. 10775-10790, Aug. 2019.

[17] Z. Ding, P. Fan, and H. V. Poor, "Impact of user pairing on 5G nonorthogonal multiple-access downlink transmissions," IEEE Trans. Veh. Technol., vol. 65, no. 8, pp. 6010-6023, Aug. 2016.

[18] R. C. Kizilirmak, C. R. Rowell, and M. Uysal, "Non-orthogonal multiple access (NOMA) for indoor visible light communications," Int. Workshop on Opt. Wirel. Commun. (IWOW), pp. 98-101, Sep. 2015.

[19] Y. Liu, Z. Ding, M. Elkashlan, and H. V. Poor, "Cooperative nonorthogonal multiple access with simultaneous wireless information and power transfer," IEEE J. Sel. Areas Commun., vol. 34, no. 4, pp. 938953, Mar. 2016

[20] Y. Ma, T. Lv, X. Li, S. Zhang, and W. Guo, "User pairing schemes in cooperative downlink NOMA system with SWIPT," in Comp., Сommun. and IoT App., Oct. 2019, pp. 82-87.

[21] M. Abd-Elnaby, G. G. Sedhom, and M. Elwekeil, "Subcarrier-user assignment in downlink NOMA for improving spectral efficiency and fairness," IEEE Access, vol. 9, pp. 5273-5284, Dec. 2020.

[22] M. T. Nguyen and L. B. Le, "NOMA user pairing and UAV placement in UAV-based wireless networks," in IEEE Int. Conf. on Commun. (ICC), May 2019, pp. 1-6.

[23] A. Alqahtani, E. A. Alsusa, A. J. Al-Dweik, and M. Al-Jarrah, "Performance analysis for downlink NOMA over $\alpha-\mu$ generalized fading channels," IEEE Trans. Veh. Technol., vol. 70, no. 7, pp. 6814-6825, May 2021.

[24] Z. Wei, D. W. K. Ng, J. Yuan, and H.-M. Wang, "Optimal resource allocation for power-efficient MC-NOMA with imperfect channel state information," IEEE Trans. Commun., vol. 65, no. 9, pp. 3944-3961, May 2017.

[25] A. A. Zavala, Indoor Wireless Communications: from Theory to Implementation. John Wiley \& Sons, Ltd, 2017.

[26] D. B. Da Costa and M. D. Yacoub, "Average channel capacity for generalized fading scenarios," IEEE Commun. Lett., vol. 11, no. 12, pp. 949-951, Dec. 2007.

[27] A. Jeffrey and D. Zwillinger, Table of Integrals, Series, and Products, 7th ed. Academic Pr, 2007.

[28] D.-T. Do and C.-B. Le, "Impact of fixed power allocation in wireless energy harvesting NOMA networks," International Journal of Communication Systems, vol. 32, pp. 1-14, Jun. 2019.

[29] M. K. Simon and M.-S. Alouini, Digital Communication Over Fading Channels, 2nd ed. New York, US: Wiley, 2004.

[30] L. Yingting, S. Jianmei, Y. Hongwu, Y. Chunman, and C. Li, "Ergodic capacity and throughput analysis of two-way wireless energy harvesting network with decode-and-forward relay," in Communications and Networking, X. Liu, D. Cheng, and L. Jinfeng, Eds. Cham: Springer Int Publishing, 2019, pp. 729-739.

[31] A. Prudnikov, Y. Brychkov, and O. Marichev, Integrals and Series. Volume 3: More Special Functions. London, UK: Godron and Breach Science, 1990.

[32] M. Springer and K. M. R. Collection, The Algebra of Random Variables. New York, US: Wiley, 1979.

[33] E. W. Weisstein. Classical meijer's integral from two G functions. [Online]. Available: http://mathworld.wolfram.com/Tree.html

[34] X. Yue, Y. Liu, S. Kang, and A. Nallanathan, "Performance analysis of NOMA with fixed gain relaying over Nakagami- $m$ fading channels," IEEE Access, vol. 5, pp. 5445-5454, Mar. 2017.

[35] J. Tang, J. Luo, M. Liu, D. K. C. So, E. Alsusa, G. Chen, K.-K. Wong, and J. A. Chambers, "Energy efficiency optimization for NOMA with SWIPT," IEEE Journal of Selected Topics in Signal Processing, vol. 13, no. 3, pp. 452-466, Feb 2019.

[36] B. Kumbhani and R. S. Kshetrimayum, MIMO Wireless Communications over Generalized Fading Channels. USA: CRC Press, Inc., 2017.

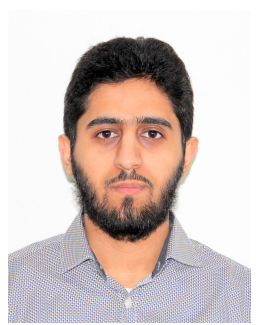

Adel Alqahtani received his M.Sc. degree in Telecommunication from George Mason University, USA, in 2017. Currently, he is a PhD candidate at the Dept. of Electrical and Electronic Engineering, The University of Manchester, U.K. His research interests include $5 \mathrm{G}$ wireless communication, NonOrthogonal multiple access (NOMA) technology, and cooperative relay networks.

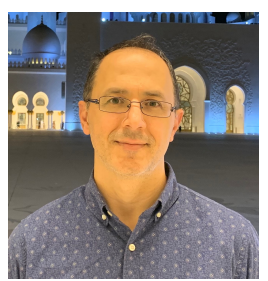

Emad Alsusa completed a $\mathrm{PhD}$ in Telecommunications from the University of Bath in the United Kingdom in 2000 and in the same year he was appointed to work on developing high data rates systems as part of an industrial project based at Edinburgh University. He joined Manchester University (then UMIST) in September 2003 as a faculty member where his current rank is a Reader in the Communication Engineering Group. His research interests lie in the area of Communication Systems with a focus on Physical, MAC and Network Layers including developing techniques and algorithms for array signal detection, channel estimation and equalization, adaptive signal precoding, interference avoidance through novel radio resource management techniques, cognitive radio and energy and spectrum optimization techniques. Applications of his research include cellular networks, IoT, Industry 4.0, and Powerline Communications. Emad's research work has resulted in over 200 journals and refereed conference publications mainly in top IEEE transactions and conferences. Emad has supervised over $30 \mathrm{PhDs}$ to successful completion. Emad is an Editor of the IEEE Wireless communication Letters, a Fellow of the UK Higher Academy of Education, and a TPC Track Chair of a number of conferences such as VTC'16, GISN'16, PIMRC'17 and Globecom'18, as well as the General Co-Chair of the OnlineGreenCom' 16 Conference. He is currently the UK representative in the International Union of Radio Science, and a Co-Chair of the IEEE Special Working Group on RF Energy Harvesting. Emad has received a number of awards including the best paper award in the international Symposium on Power Line Communications 2016 and the Wireless Communications and Networks Conference 2019.

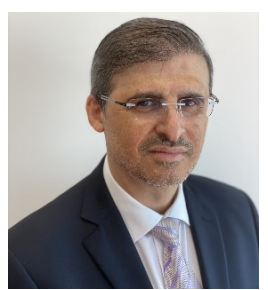

Arafat Al-Dweik received the M.S. (Summa Cum Laude) and Ph.D. (Magna Cum Laude) degrees in electrical engineering from Cleveland State University, Cleveland, OH, USA, in 1998 and 2001, respectively.

He is currently with the Department of Electrical Engineering and Computer Science, Khalifa University, Abu Dhabi, UAE. He also worked at Efficient Channel Coding, Inc., Cleveland, OH, USA, Department of Information Technology, Arab American University, Jenin, Palestine, and University of Guelph, ON, Canada. He is a Visiting Research Fellow with the School of Electrical, Electronic, and Computer Engineering, Newcastle University, Newcastle upon Tyne, U.K, and a Research Professor with Western University, London, ON, Canada, and University of Guelph, Guelph, Canada. He has extensive research experience in various areas of wireless communications that include modulation techniques, channel modeling and characterization, synchronization and channel estimation techniques, OFDM technology, error detection and correction techniques, MIMO, and resource allocation for wireless networks.

Dr Al-Dweik serves as an Associate Editor for the IEEE Transactions on Vehicular Technology and the IET Communications. He is a member of Tau Beta Pi and Eta Kappa Nu. He was awarded the Fulbright scholarship from 1997 to 1999. He was the recipient of the Hijjawi Award for Applied Sciences in 2003, Fulbright Alumni Development Grant in 2003 and 2005, Dubai Award for Sustainable Transportation in 2016, UAE Leader-Founder Award in 2019. $\mathrm{He}$ is a Registered Professional Engineer in the Province of Ontario, Canada. 